\title{
Bildungspolitik: Nicht alles anders, aber manches
}

\section{Marius R. Busemeyer und Susanne Haastert}

\section{Zusammenfassung}

Dieses Kapitel beschreibt und diskutiert die Bildungsreformen der grün-roten Landesregierung. Nach einer kurzen Einführung in die wichtigsten theoretischen Perspektiven dokumentieren wir zunächst den bildungspolitischen Status Quo im Jahr 2011 vor Antritt der grün-roten Landesregierung und zeigen, dass es in Baden-Württemberg durchaus bildungspolitischen Handlungsbedarf gab, insbesondere bei der Reform der Schulstrukturen und der Inklusion. In drei Fallstudien zu wichtigen Reformprojekten (die Abschaffung der Verbindlichkeit der Grundschulempfehlung, die Einführung der Gemeinschaftsschule und die Umsetzung des Inklusionsprinzips in Schulen) wird deutlich, dass die grün-rote Landesregierung diese Kernprojekte im Großen und Ganzen auch gegen den Widerstand der Opposition umsetzen konnte. In einer abschließenden Bewertung und Analyse argumentieren wir, dass die wesentlichen Kernelemente dieser Reformen auch langfristig Bestand haben werden, da sie über die Erweiterung von Elternwahlrechten und die Mobilisierung von lokalen Akteuren im Rahmen von konsensorientierten Strukturreformen wirkmächtige Feedback-Effekte erzeugen.

M.R. Busemeyer $(\bowtie) \cdot$ S. Haastert

Fachbereich Politik und Verwaltungswissenschaft, Universität Konstanz, Konstanz,

Deutschland

E-Mail: marius.busemeyer@uni-konstanz.de

S. Haastert

E-Mail: susanne.haastert@uni-konstanz.de 


\section{$1 \quad$ Einleitung}

Im bundesdeutschen Föderalismus verbleibt den Bundesländern im Bereich der Bildungspolitik ein erheblicher Gestaltungsspielraum, insbesondere bei der Ausgestaltung der Schulpolitik - dem „Kernbestand der Kulturhoheit“ (Wolf 2008). Durch die Föderalismusreform im Jahr 2006 wurde die Kompetenzhoheit der Bundesländer in der Bildungspolitik weiter gestärkt, wenngleich dies vor allem den Bereich der Hochschulpolitik und weniger die allgemeinbildenden Schulen betraf, denn hier hatten die Länder auch schon vor der Reform weitreichende Kompetenzen. So entscheiden die Bundesländer beispielsweise über die Ausprägung der Schulstrukturen, die Höhe und die Verteilung der Bildungsausgaben, die Bildungsplaninhalte, die Standardisierung der Abschlussprüfungen und die Ausgestaltung der Lehrerbildung.

Auch wenn der Gestaltungsfreiheit der Länder durch institutionalisierte Mechanismen der horizontalen Koordination, insbesondere die Kultusministerkonferenz (KMK), faktische Grenzen auferlegt sind, hat die Hoheit der Bundesländer in der Ausgestaltung der Bildungspolitik dennoch zur Entstehung einer vielfältigen Bildungslandschaft beigetragen (vgl. Wolf 2006, 2008; Von Below 2002; zum Folgenden insbes. Helbig und Nikolai 2015, Abschn. 4.1). So ist in den meisten westdeutschen Flächenländern zusätzlich zum klassischen dreigliedrigen System (Gymnasium, Realschule, Hauptschule) mindestens ein weiterer Schultypus (Gesamtschule und/oder andere Schularten mit mehreren Bildungsgängen) hinzukommen, sodass hier von vier- oder fünfgliedrigen Systemen gesprochen werden muss (in Nordrhein-Westfalen, Hessen und Niedersachsen). Die ostdeutschen Flächenländer und die Stadtstaaten (Bremen, Berlin und Hamburg), aber auch Rheinland-Pfalz und das Saarland, sind zu unterschiedlichen Zeitpunkten und in unterschiedlicher Form zu strukturell zweigliedrigen Systemen übergegangen, in denen neben dem Gymnasium nur noch eine weitere Schulform auf Sekundarschulebene existiert - in der Regel eine Schulart, die mehrere Bildungsgänge miteinander vereint, in verschiedenen Bundesländern aber auch unterschiedlich bezeichnet wird (die „Realschule Plus“ in RheinlandPfalz, die „Stadtteilschule“ in Hamburg oder die „Gemeinschaftsschule“ im Saarland, in Thüringen und in Schleswig-Holstein). Diese großen Unterschiede zwischen den Schulstrukturen verschiedener Bundesländer verdeutlichen, dass die Landesregierungen im Bereich der Bildungspolitik durchaus einen beachtlichen Gestaltungs- und Handlungsspielraum haben, der größer ist als in vielen anderen Politikfeldern.

In diesem Kapitel möchten wir vor diesem Hintergrund verschiedene Forschungsfragen beantworten: Wie kann der status quo ante in der 
baden-württembergischen Bildungspolitik vor Antritt der grün-roten Landesregierung charakterisiert werden? Welche bildungspolitischen Reformen hat die grünrote Landesregierung verfolgt und umgesetzt, um diesen status quo zu verändern? Stellen diese Reformen lediglich inkrementelle Veränderungen dar, oder sind langfristig transformative Auswirkungen auf die institutionelle Ausgestaltung des baden-württembergischen Schulsystems zu erwarten?

Zur Struktur des vorliegenden Kapitels: Im nächsten Abschnitt werden wir zunächst kurz theoretische Überlegungen skizzieren, ob bzw. unter welchen Bedingungen der Regierungswechsel zu Grün-Rot mit einem signifikanten Politikwandel in der Bildungspolitik einhergehen sollte. Im anschließenden empirischen Teil werden wir zunächst den bildungspolitischen status quo ante vor Antritt der grün-roten Landesregierung im Jahr 2011 und die Positionen der einzelnen Parteien zu diesem status quo ante anhand ihrer Wahlprogramme skizzieren. Anschließend geben wir einen Überblick über die Reformen im Bildungswesen und untersuchen drei der wichtigsten Bildungsreformen in kurzen Fallstudien. Wir schließen mit einer Gesamtbewertung zur Frage, ob die grünroten Bildungsreformen einen langfristigen und politisch nachhaltigen Prozess des institutionellen Wandels im baden-württembergischen Schulsystem eingeleitet haben.

\section{Theoretische Vorüberlegungen}

Die Thematik, ob Regierungswechsel systematisch mit Policy-Wandel einhergehen, ist eine zentrale Frage der vergleichenden Policy-Forschung. Auf der einen Seite stehen Anhänger der Parteiendifferenztheorie (vgl. Castles 1982; Schmidt 1980, 1996, 2002), die diese Frage positiv beantworten. Die Parteiendifferenztheorie postuliert, dass sich die Policy-Präferenzen politischer Parteien deswegen systematisch unterscheiden, weil sie unterschiedliche Wählerklientel repräsentieren. Die klassische Variante der Parteiendifferenztheorie führte die politischen Interessen und Forderungen dieser Klientelgruppen auf unterschiedliche ökonomische Interessen entlang der Markt-Staat-Achse zurück (Castles 1982; Schmidt 1980). Linke Parteien sind demnach sozialstaatsfreundlicher, weil sie die Interessen der unteren Einkommensschichten vertreten, während rechte bzw. bürgerliche Parteien die wohlhabenderen Schichten repräsentieren und deswegen eher auf niedrigere Steuern statt einen weiteren Ausbau des Sozialstaates setzen. Neuere Varianten der Parteiendifferenztheorie erweitern diese Perspektive aufgrund der Tatsache, dass sich der parteipolitische Wettbewerb inzwischen nicht mehr ausschließlich auf der ökonomischen Markt-Staat-Achse abspielt, sondern auch auf 
einer zweiten Dimension, bei der Wertekonflikte eine größere Rolle spielen (vgl. grundlegendend Kitschelt 1994; sowie Häusermann et al. 2013). Den einen Pol dieser zweiten Achse bilden liberale Wertvorstellungen, wie zum Beispiel Unterstützung für Globalisierung, ein egalitäres Geschlechterverhältnis oder Umweltschutz. Im Gegensatz dazu finden sich am anderen Pol konservativ-autoritäre Wertvorstellungen, die stärker traditionelle Familienbilder und paternalistischhierarchische Sozialstrukturen betonen.

Wie vor allen Dingen die Arbeiten von Kitschelt $(1994,1999)$ gezeigt haben, stellt diese Rekonfiguration des politischen Raums vor allem für sozialdemokratische Parteien ein Dilemma dar. Einerseits müssen sie versuchen, durch ihre Politikangebote weiterhin ihre klassischen Klientel in den unteren Einkommensschichten und im Arbeitermilieu zu bedienen. Anderseits müssen angesichts des demografisch und sozio-strukturell bedingten Niedergangs des klassischen Arbeitermilieus auch neue Wählergruppen in der Mittelschicht angesprochen werden, die im Gegensatz zum Arbeitermilieu auf der zweiten, werteorientierten Achse dem liberalen Pol näher stehen (Gingrich und Häusermann 2015). Grüne Parteien waren und sind diesem Dilemma in weit weniger starkem Maße ausgesetzt. Aufgrund ihrer politischen Wurzeln in den außerparlamentarischen sozialen Bewegungen der 1960er und 1970er Jahre, die letztlich auch durch die Dynamik des gesellschaftlichen Wertewandels hin zum Post-Materialismus befördert wurden, nehmen grüne Parteien auf der liberal-autoritären Achse eine stärker homogene Position nahe des liberalen Pols ein. In Deutschland hat sich die grüne Partei zudem auf der Markt-Staat-Achse klar auf der linken Seite positioniert, auch wenn die baden-württembergischen Grünen im Vergleich zu anderen Landesverbänden hier eher Mitte-orientiert sind (Schlauch und Weber 2015).

Bezogen auf den Gegenstand der Bildungspolitik könnte daher erwartet werden, dass sich die grün-rote Regierungspolitik deutlich von der Politik der Vorgängerregierung unterscheidet. Die einschlägige Forschung zu Parteieneffekten in der Bildungspolitik hat argumentiert und gezeigt, dass sozialdemokratische Parteien tendenziell mehr für Bildung ausgeben, sich für Strukturreformen in Richtung eines Gemeinschafts- oder Gesamtschulmodells einsetzen, eher staatliche statt marktliche Steuerungsformen präferieren und sich für eine Ausweitung des Zugangs zu den höheren Stufen des Bildungswesens einsetzen (Ansell 2008; Boix 1997; Busemeyer 2007, 2009, 2015; Gingrich 2011; Nikolai 2007; Schmidt 1980, 2007; Wolf 2006; Wolf und Zohlnhöfer 2009).

$\mathrm{Zu}$ den bildungspolitischen Positionen von grünen Parteien gibt es wenig bis gar keine einschlägige Forschung. Dies mag auch daran liegen, dass grüne Parteien im internationalen Vergleich in der Bildungspolitik in vielen Ländern als „,issue ignorer“ (Busemeyer et al. 2013) auftreten, d. h. dem Thema insgesamt 
eine eher geringe Aufmerksamkeit widmen, da sie stärker mit anderen Themen wie etwa Umweltschutz oder Geschlechterpolitik befasst sind. Ein weiterer Grund ist schlicht die Tatsache, dass grüne Parteien bisher immer lediglich als kleiner Koalitionspartner an Regierungen beteiligt waren. Als Ausgangshypothese, der wir in den Fallstudien weiter nachgehen möchten, kann hier lediglich festgehalten werden, dass die Partei der Grünen - insbesondere in Deutschland als links-liberale Partei - in wesentlichen Punkten ein ähnliches Policy-Profil aufweisen sollte wie sozialdemokratische Parteien. Wenn diese These zutreffen würde (oder die Grünen im Vergleich zur SPD sogar noch weiter „links“ zu verorten wären), würde dies allerdings wesentliche Grundannahmen der klassischen Parteiendifferenztheorie herausfordern: Die Wählerklientel der Grünen sind tendenziell wohlhabender als die der SPD und sollten insofern näher an den bildungspolitischen Positionen bürgerlicher Parteien sein. Wenn dem nicht so wäre, würde dies darauf hindeuten, dass die Werte-Dimension - zumindest bei den Wähler*innen der Grünen - eine gewichtigere Rolle spielt als die ökonomische Dimension.

Im Gegensatz dazu ist zu erwarten, dass die ehemaligen Oppositionsparteien, insbesondere die CDU, als Verteidiger des gegliederten Schulsystems auftreten und weniger hohe Bildungsausgaben anstreben. Christdemokratische Bildungspolitik begründet die Notwendigkeit eines gegliederten bzw. differenzierten Schulsystems damit, dass auf diese Weise unterschiedliche Begabungen und Fertigkeiten besser gefördert werden als durch ein Bildungssystem, das durch die Einführung neuer Schultypen wie der Gesamtschule diese inhärenten Unterschiede nicht Ernst genug nehme (vgl. etwa Wilms 1986).

Wolf (2006) konnte in einer umfassenden Analyse der Bildungsausgabenpolitik der Länder den erwarteten Zusammenhang zwischen der parteipolitischen Färbung einer Landesregierung und den Bildungsausgaben feststellen; in der späteren Zeitperiode scheinen es jedoch vor allem CDU/CSU-geführte Regierungen gewesen zu sein, die mehr für Bildung ausgegeben haben (vgl. auch Rauh et al. 2011 für den Bereich der Hochschulpolitik). Abgesehen von der Frage der Bildungsfinanzierung kristallisieren sich parteipolitische Konflikte zwischen den beiden großen Parteien über die institutionelle Ausgestaltung von Schulstrukturen weiterhin an der Frage, wie bzw. ob das gegliederte Sekundarschulwesen reformiert werden soll (Edelstein und Nikolai 2013; Helbig und Nikolai 2015). Neben der politischen Debatte zur Schulstruktur, die nun bereits seit Jahrzehnten geführt wird, sind allerdings auch neue Themen auf die bildungspolitische Agenda gekommen, insbesondere die Problematik der Umsetzung des Inklusionsprinzips an Schulen in Folge der Verabschiedung der UN-Behindertenrechtskonvention im Jahr 2009 (Blanck et al. 2014), die ebenfalls zwischen den Parteien umstritten 
sind. Tendenziell sind hier linke Parteien stärker engagiert als bürgerliche Parteien, da sie das Ziel der Bildungsgerechtigkeit höher gewichten.

Auch wenn sich die Parteiendifferenzthese in vielen Politikfeldern als erklärungskräftig erwiesen hat, gibt es gewichtige Einwände gegen ihre Gültigkeit. Im vorliegenden Beitrag möchten wir vor allen Dingen auf den potenziellen Einfluss des institutionellen Politikerbes eingehen. Wie Pierson (1993, 1994, 1996) und andere (Rose und Davies 1994) gezeigt haben, haben bestehende Institutionen einen starken Einfluss auf die Größe des reformpolitischen Spielraums. Verschiedene politische Akteure (organisierte Interessen, aber auch individuelle Bürger*innen) haben ihre Strategien und Präferenzen an den bestehenden Institutionen ausgerichtet. Diese „Policy-Feedback-Effekte“ führen dazu, dass der Widerstand gegen weitreichende systemische/strukturelle Reformen in institutionalisierten Bildungs- oder wohlfahrtsstaatlichen Regimen groß ist. In der deutschen Bildungspolitik beispielsweise sind die Berufsverbände, die die Interessen der Lehrer*innen vertreten, in weiten Teilen auf der Grundlage der unterschiedlichen Schultypen des gegliederten Schulsystems organisiert. Allein aus diesem Grund ist zu erwarten, dass Reformversuche, die auf eine vollständige Überwindung des gegliederten Schulsystems zielen würden, auf großen Widerstand stoßen werden.

In seinen Arbeiten zum Wohlfahrtsstaat betont Pierson (1993, 1994, 2001) vor allen Dingen die Tatsache, dass bestehende Institutionen den Spielraum für Veränderungen aufgrund der soeben skizzierten ,positiven“ (also: den Status quo bestärkenden) Feedback-Effekte und der damit einhergehende Pfadabhängigkeiten beschränken. Im Gegensatz dazu haben neuere Arbeiten in der Tradition des historischen Institutionalismus allerdings herausgearbeitet, dass diese „positiven“ unter bestimmten Bedingungen auch in „,negative“ (also: den Status quo verändernden) Feedback-Effekte umschlagen können. Zudem muss eine solche Transformation nicht zwangsläufig durch exogene Faktoren ausgelöst werden, sondern kann auch Resultat systemimmanenter, also endogener Veränderungsprozesse sein (vgl. etwa Greif und Laitin 2004; Jacobs und Weaver 2015; Soroka und Wlezien 2010; Streeck und Thelen 2005; Weaver 2010 sowie Busemeyer und Trampusch 2013 für eine Anwendung auf den deutschen Fall). Insofern können bestehende Institutionen aus sich selbst heraus auch politische Unterstützung für eine Abkehr vom Status Quo generieren, wenn dieser Status Quo etwa Nebeneffekte produziert, die aus der Perspektive einer relativen Mehrheit der Betroffenen negativ zu bewerten sind.

Im Folgenden untersuchen wir, inwiefern die Theorien der Parteiendifferenz, der Pfadabhängigkeit und der Feedback-Effekte die Parteipositionen und Bedingungen für Politikwandel in Baden-Württemberg 2011 erklären können. 


\section{Ausgangslage und Parteipositionen vor dem Regierungswechsel}

Um die Vielzahl an Reformen einordnen zu können, ist zunächst ein Blick auf die Ausgangslage im Jahr 2011 hilfreich. Verschiedene Punkte sind hier relevant. Erstens war aufgrund des demografischen Wandels und sinkenden Schülerzahlen auf längere Frist ein flächendeckendes Angebot von Abschlussmöglichkeiten nicht mehr gewährleistet. Infolge des Akzeptanzverlusts der Haupt-/Werkrealschulen $^{1}$ sanken die Anmeldungen insbesondere an dieser Schulart, und viele Schulen mussten bereits schließen oder waren von der Schließung bedroht (Landesinstitut für Schulentwicklung 2015, S. 104-106).

Zweitens war in Baden-Württemberg im Vergleich zu anderen Bundesländern der Bildungserfolg von Kindern relativ stark von der sozialen Herkunft abhängig (Köller et al. 2010, S. 22). Dies lässt sich laut Bildungsforschern auch auf das gegliederte Schulsystem zurückführen (Hanushek und Wößmann 2006; Pfeffer 2008). So war Baden-Württemberg eines der wenigen Bundesländer ohne signifikante Anzahl an Schüler*innen in integrierten Gesamtschulen oder anderen Schulformen mit mehreren Bildungsgängen (Autorengruppe Bildungsberichterstattung 2012, S. 69). Im Schuljahr 2012/2013 beispielsweise besuchten lediglich 2,7 \% der Fünftklässler in Baden-Württemberg eine Integrierte Gesamtschule ${ }^{2}$ bei einem deutschlandweiten Durchschnitt von 24,8 \% (Autorengruppe Bildungsberichterstattung 2014, S. 254). Nur Bayern (0,3\%) lag noch dahinter.

Drittens hatte Baden-Württemberg einen vergleichsweise niedrigen Anteil an Ganztagsschulen. Während der Bundesdurchschnitt bei $51 \%$ lag, hatten in Baden-Württemberg lediglich $27 \%$ der Schulen Ganztagsangebote (Autorengruppe Bildungsberichterstattung 2012, S. 79). Angesichts gestiegener örtlicher Mobilität und der Veränderung von Familienstrukturen leisten ganztägige Betreuungsangebote einen wichtigen Beitrag zur Vereinbarkeit von Familie und Beruf und erleichtern die Beteiligung von Frauen im Arbeitsmarkt. Insbesondere für Alleinerziehende sind Ganztagsschulen wichtig, um ihnen die Ausübung einer

\footnotetext{
${ }^{1}$ Werkrealschulen sind eine Weiterentwicklung der Hauptschulen, die durch einen Landtagsbeschluss unter der CDU-FDP-Regierungskoalition im Jahr 2009 gesetzlich vorgeschrieben wurde. Werkrealschulen bieten nach dem Hauptschulabschluss zusätzlich ein zehntes Schuljahr an um Hauptschüler*innen mehr Abschlussmöglichkeiten zu bieten (Schulgesetz § 6).

${ }^{2}$ Oder ein Sport- und Musikgymnasium. Diese beiden Schulformen werden von den Autoren des Bildungsberichts 2014 zusammengelegt.
} 
Vollzeittätigkeit zu erleichtern. Zudem deuten Studienergebnisse an, dass Ganztagsschulen sich positiv auf die Fähigkeiten und Kompetenzen insbesondere von Kindern aus bildungsferneren Familien auswirken; qualitativ hochwertige Ganztagsangebote können somit auch zur Entkoppelung des Zusammenhangs von sozialer Herkunft und Bildungserfolg beitragen (Expertenrat „Herkunft und Bildungserfolg“ 2011, S. 14, 90).

Schließlich lag Baden-Württemberg in Bezug auf die Umsetzung von Inklusion aufgrund von Schulversuchen zum Zeitpunkt des Regierungswechsels bereits über dem Bundesdurchschnitt. So wurden im Schuljahr 2010/11 in BadenWürttemberg 27,4\% aller Schüler*innen mit sonderpädagogischem Förderbedarf in allgemeinbildenden Schulen unterrichtet, während es bundesweit erst 22,4 \% waren (Autorengruppe Bildungsberichterstattung 2012, Tab. D1-4 A). Jedoch beruhte dieser vergleichsweise hohe Wert teilweise auf der Erprobung von Inklusion in fünf zum Schuljahr 2010/2011 eingeführten Modellregionen sowie auf inklusiven Angeboten in Form von Außenklassen (Kultusministerium 2010). In Außenklassen kooperiert eine Sonder- oder Förderschule mit einer allgemeinbildenden Schule und bietet teilweise gemeinsamen Unterricht an. Diese Art der Inklusion wird jedoch kritisiert, da sie keine innerschulische Inklusion darstellt (Preuss-Lausitz 2010). Zudem war das Recht von Menschen mit Behinderung auf Bildung an allgemeinbildenden Schulen nicht im Schulgesetz verankert. Insofern bestand auch im Themenfeld Inklusion ein gewisser Handlungsbedarf.

Mit der Theorie der Feedback-Effekte lässt sich angesichts dieser Herausforderungen sagen, dass es neben starken positiven Feedback-Effekten aufgrund der lang etablierten Schulstruktur und entsprechend organisierter Interessengruppen auch beträchtliche negative Feedback-Effekte gab. So verursachte offensichtlich die Aufrechterhaltung eines differenzierten, gegliederten Schulwesens in ländlichen Räumen zunehmend finanzielle und andere praktische Schwierigkeiten, und die seit PISA geführte, öffentliche Diskussion über Bildungsgerechtigkeit stellte das gegliederte Schulsystem zunehmend infrage. Diese Gründe könnten nicht nur dazu beigetragen haben, dass die von Grün-Rot angestrebten Bildungsreformen politisch umsetzbar(er) wurden, sondern auch dazu, dass sich die Wähler*innen am 27. März 2011 überhaupt gegen die alte und für eine neue Regierung entschieden hatten. Doch: Wie planten die größten Parteien, diese Herausforderungen anzugehen?

Aus Sicht der CDU gab es keinen Grund die Schulstrukturen zu verändern. Den demografischen Wandel wollte die CDU hauptsächlich durch Familienförderung und verstärkte Einbindung älterer Menschen in den Arbeitsmarkt angehen (CDU 2011a, S. 40-43). Die CDU sah darüber hinaus vor, die Ganztagsschulen je nach Nachfrage vor Ort weiter auszubauen (CDU 2011a, S. 43). In Bezug auf 
die weitere Umsetzung von Inklusion findet sich im Wahlprogramm der CDU keine Aussage; eine Gesetzesänderung war jedoch zum Schuljahr 2013/2014 geplant (Rodenhausen 2010). Auch die FDP sah keine strukturellen Veränderungen im Schulwesen vor. Im Gegensatz zur CDU setzte sich die FDP allerdings für die Ermöglichung integrativer und innovativer Schulprojekte und -kooperationen ein (Rodenhausen 2010, S. 36-40) und beschrieb konkrete Maßnahmen zur Umsetzung von Inklusion, wie z. B. die Öffnung aller Schularten für Kinder mit und ohne Behinderung und die Verankerung des beschränkten Elternwahlrechts ${ }^{3}$ (Rodenhausen 2010, S. 58 f.).

Für die SPD war Bildung das zentrale Wahlkampfthema. Sie beschrieb das gegliederte Schulsystem als Hindernis sowohl für soziale Gerechtigkeit als auch für den Erhalt von Schulstandorten, insbesondere im ländlichen Raum, und plante daher, im Dialog mit den Beteiligten vor Ort eine Schulstrukturreform umzusetzen. Diese sollte in der Einführung einer 10-jährigen Gemeinschaftsschule (mit anschließender gymnasialer Oberstufe) münden (SPD 2011, S. 16 f.). Neben der geplanten Schulstrukturreform versprach die SPD, verlässliche Rahmenbedingungen für Ganztagsschulen zu schaffen und die Umsetzung von Inklusion im Schulgesetz zu verankern (ohne hierfür konkrete Maßnahmen vorzuschlagen) (SPD 2011, S. 18-21).

Auch die Grünen setzten sich für eine 10-jährige gemeinsame Lernzeit an einer Basis- oder Gemeinschaftsschule ein. Dabei betonten sie, dass Kommunen und Gesellschaft mehr Verantwortung bei der Gestaltung der Schulstruktur bekommen sollten (Die Grünen 2011a, S. 98-107). Zudem versprachen die Grünen, Ganztagsschulen schneller auszubauen, indem zusätzliche Lehrerstellen bereit gestellt werden sollten (Die Grünen 2011a, S. 107 f.). In Bezug auf die Umsetzung von Inklusion befürworteten die Grünen ebenso wie die FDP eine Öffnung aller Schularten für Kinder mit und ohne Behinderung (Die Grünen 2011a, S. 109 f.). Jedoch gingen die Vorhaben der Grünen darüber hinaus: Sie wollten die Sonderschulpflicht sofort abschaffen und ein uneingeschränktes Elternwahlrecht im Schulgesetz verankern (Die Grünen 2011a, S. 109 f.). Als einzige Partei gingen die Grünen auch auf die anstehende Bildungsplanreform ein und forderten, ein Leitbild der nachhaltigen Entwicklung - Umweltbildung, Gleichberechtigung der Geschlechter, Toleranz und Gerechtigkeit - als roten Faden in die Bildungspläne zu integrieren (Die Grünen 2011a, S. 116 f.).

\footnotetext{
${ }^{3}$ Von einem ,beschränkten“ Elternwahlrecht wird gesprochen, wenn die zuständige Behörde unter Angabe überzeugender Gründe eine Schulform wählen kann, die nicht dem Elternwillen entspricht.
} 
Dieser Überblick über die Parteipositionen zu den wichtigsten Herausforderungen zeigt, dass die Erwartungen der Parteidifferenztheorie weitgehend zutreffen: CDU und FDP versprachen - im Sinne ihrer konservativeren Wähler aus höheren Einkommens- und Bildungsschichten - den Erhalt des dreigliedrigen Schulsystems. Die SPD setzte sich für eine Schulstrukturreform zugunsten eines Gesamtschulsystems ein, was den Kindern von Arbeiterfamilien neue und erleichterte Zugangsmöglichkeiten zu höheren Bildungsabschlüsse bieten könnte. Die Grünen legten ein besonderes Augenmerk auf Ganztagsbetreuung und Beteiligungsprozesse, wovon ihre tendenziell höher gebildeten und politisch aktiveren Wähler direkt profitieren. Zudem legten sie großen Wert auf die Ausgestaltung von Bildungsinhalten und Betreuungskonzepten, soziale Gerechtigkeit und Inklusion, welche sie u. a. durch die Gemeinschaftsschule erreichen wollten. Hier ist ein Paradox zu erkennen: Aufgrund der höheren Bildungsabschlüsse der Grünen-Wähler könnte man annehmen, dass diese - aufgrund von materialistischen Überlegungen - eine Förderung von Gymnasium und Hochschulbildung vorziehen, aber die Einführung einer Gemeinschaftsschule ablehnen müssten. Wie oben bereits angedeutet scheint hier die postmaterialistische Orientierung der Grünen und ihrer Wählerklientel dazu beizutragen, dass die Partei sich für eine Schulform einsetzt, die nicht unbedingt den materiellen Interessen ihrer Wählerschaft entspricht, wohl aber ideellen Überzeugungen.

Zusammenfassend betrachtet waren die Ausgangsbedingungen für ambitiöse grün-rote Bildungsreformen bei dem Machtwechsel im Jahr 2011 daher nicht ungünstig. Die Policy-Positionen der beiden Koalitionspartner waren in diesem Politikfeld weit weniger weit voneinander entfernt als in anderen (wie etwa dem Konflikt um „Stuttgart 21"). Auch wenn die politischen und institutionellen Beharrungskräfte des bestehenden Bildungssystems durchaus beträchtlich waren, gab es einige Anhaltspunkte für Defizite im baden-württembergischen Bildungswesen, die letztlich auch auf den mangelnden Reformeifer der Vorgänger-Regierungen zurückgeführt werden konnten. Im folgenden Abschnitt befassen wir uns damit, welche Reformprojekte die grün-rote Landesregierung umsetzte und zeigen anhand einiger Fallbeispiele zu den wichtigsten grün-roten Reformprojekten, dass die Umsetzung der bildungspolitischen Reformen trotz vergleichbar günstiger Ausgangslage politisch recht kontrovers verlief. 


\section{4 Überblick über die Bildungsreformen der grün- roten Landesregierung}

Mit dem Koalitionsvertrag einigten Grüne und SPD sich auf bestimmte, konkrete Reformvorhaben im Bildungsbereich, zum Beispiel die verbindliche Grundschulempfehlung durch eine Elternberatung $\mathrm{zu}$ ersetzen, Gemeinschaftsschulen auf Wunsch von Schulen und Schulträgern zu ermöglichen und zu fördern, den Anspruch auf Inklusion ins Schulgesetz aufzunehmen und das Elternwahlrecht einzuführen, sowie „Bildung für nachhaltige Entwicklung“ als roten Faden in den Bildungsplänen zu verankern (Die Grünen und SPD 2011, S. 5-8). Im Vergleich mit den Wahlprogrammen der Parteien lässt sich erkennen, dass sich im Bereich der Inklusion ebenso wie in Bezug auf die Bildungspläne die expliziteren Vorstellungen der Grünen durchgesetzt haben. Die Begrifflichkeiten und Vorhaben der SPD dominieren dagegen das Themenfeld der Gemeinschaftsschulen - und damit den Bereich möglicher Schulstruktur-Reformen.

Ebenfalls einigten sich die Koalitionäre darauf, dass das Kultusministerium von SPD-Mitglied Gabriele Warminski-Leitheußer geleitet werden sollte. Während der Legislaturperiode initiierte die grün-rote Landesregierung eine Vielzahl an Reformen (siehe Tab. 6) im Bildungswesen, die teilweise höchst kontrovers diskutiert wurden. Jedoch war die Bildungspolitik nicht nur von vielen Reformen, sondern auch von einem Wechsel an der Spitze des Kultusministeriums geprägt: Anfang Januar 2013 gab Kultusministerin Warminski-Leitheußer bekannt, dass sie ihr Amt aufgrund fehlenden Rückhalts in der eigenen Landtagsfraktion niederlegte (Kultusministerium 2013a). Als weitere Gründe für den Rücktritt gelten ihre schwierige Situation als SPD-Ministerin in einem jahrzehntelang CDU-geführten Haus sowie mangelnde Kommunikationsfähigkeit und Überforderung (Exp11; Wieselmann 2013). Am 23. Januar wurde Andreas Stoch als neuer Kultusminister im Amt vereidigt (Kultusministerium 2013b). Er bemühte sich in der Folge sehr, die Kommunikation mit den relevanten Stake-Holdern zu verstärken und die Bildungspolitik insgesamt ruhiger und sachlicher anzugehen (Exp11; Exp18). Tab. 6 gibt in chronologischer Reihenfolge einen Überblick über die wichtigsten Reformen unter Grün-Rot im Bildungssystem.

Neben den Reformen, die wir im folgenden Abschnitt im Rahmen von Fallstudien eingehender untersuchen, trug insbesondere die Überarbeitung der Bildungspläne zu kontroversen Diskussionen bei. Eine solche Überarbeitung findet etwa alle zehn Jahre statt und stand während der Legislaturperiode der grün-roten 
Tab. 6 Die wichtigsten Bildungsreformen im Überblick, März 2011 - März 2016

\begin{tabular}{|c|c|c|}
\hline Datum & Reform & Inhalte der Reform \\
\hline 01.12 .2011 & Pakt für Familien mit Kindern & $\begin{array}{l}\text { Das Land verpflichtet sich, seine } \\
\text { Beiträge zur Finanzierung der } \\
\text { Kleinkindbetreuung auf } 68 \% \text { der } \\
\text { Betriebsausgaben zu erhöhen, um } \\
\text { die Kommunen beim Ausbau von } \\
\text { Betreuungsplätzen für Kleinkinder } \\
\text { zu unterstützen }\end{array}$ \\
\hline 17.12 .2011 & $\begin{array}{l}\text { Abschaffung der Verbindlichkeit der } \\
\text { Grundschulempfehlung; } \\
\text { Schulgesetz § 5(2) }\end{array}$ & $\begin{array}{l}\text { Die Grundschulen haben die } \\
\text { Aufgabe, die Eltern umfassend zu } \\
\text { beraten, welche Schulart für ihr Kind } \\
\text { geeignet ist; die Entscheidung treffen } \\
\text { jedoch die Eltern }\end{array}$ \\
\hline 21.04 .2012 & $\begin{array}{l}\text { Einführung der Gemeinschaftsschule; } \\
\text { Schulgesetz § 8a }\end{array}$ & $\begin{array}{l}\text { Die Gemeinschaftsschule ist eine } \\
\text { Ganztagsschule und inklusive } \\
\text { Schule. Schüler*innen lernen in eis- } \\
\text { tungsheterogenen Gruppen gemein- } \\
\text { sam bis zur 10. Klasse und arbeiten } \\
\text { je nach individuellem Leistungsstand } \\
\text { auf dem Niveau der Hauptschule, der } \\
\text { Realschule oder des Gymnasiums. } \\
\text { Sie bekommen verbales und schrift- } \\
\text { liches Feedback; Noten werden in } \\
\text { der Abschlussklasse erteilt }\end{array}$ \\
\hline 22.05 .2014 & $\begin{array}{l}\text { Festlegung eines Verfahrens für die } \\
\text { regionale Schulentwicklung; } \\
\text { Schulgesetz } § 30\end{array}$ & $\begin{array}{l}\text { Das Verfahren der regionalen Schul- } \\
\text { entwicklung wird auf Antrag eines } \\
\text { Schulträgers, Landkreises oder einer } \\
\text { Gemeinde und bei Unterschreitung } \\
\text { einer Mindestschülerzahl eingelei- } \\
\text { tet. Alle „Berührten“ sind an dem } \\
\text { Verfahren zu beteiligen, insbes. } \\
\text { weitere Schulen und Gemeinden } \\
\text { im relevanten Gebiet (Raumschaft). } \\
\text { Wird kein Konsens erreicht, kommt } \\
\text { es zu einer Schlichtung durch die } \\
\text { obere Schulaufsichtsbehörde }\end{array}$ \\
\hline 16.04 .2014 & $\begin{array}{l}\text { Schulganztag in Grundschulen \& } \\
\text { Grundstufen der Förderschulen; } \\
\text { Schulgesetz § } 4\end{array}$ & $\begin{array}{l}\text { Ganztagsschulen bieten an drei/vier } \\
\text { Tagen der Woche eine pädagogisch } \\
\text { sinnvolle Verbindung von Unterricht, } \\
\text { Übungsphasen, Förderzeiten, Aktiv- } \\
\text { und Kreativzeiten an und binden } \\
\text { dabei außerschulische Partner ein }\end{array}$ \\
\hline
\end{tabular}


Tab. 6 (Fortsetzung)

\begin{tabular}{|c|c|c|}
\hline Datum & Reform & Inhalte der Reform \\
\hline 15.06 .2015 & $\begin{array}{l}\text { Umsetzung von Inklusion; } \\
\text { Schulgesetz } § 15\end{array}$ & $\begin{array}{l}\text { Inklusion von Kindern mit Anspruch } \\
\text { auf sonderpädagogische Förderung } \\
\text { ist Aufgabe aller Schulen. Nach } \\
\text { umfassender Beratung auf einer } \\
\text { Bildungswegekonferenz können die } \\
\text { Erziehungsberechtigten entscheiden, } \\
\text { ob ihr Kind in einer allgemeinen } \\
\text { Schulen oder einem Sonderpädago- } \\
\text { gischen Bildungs- und Beratungs- } \\
\text { zentren (SBBZ) unterrichtet werden } \\
\text { soll }\end{array}$ \\
\hline 30.09 .2015 & $\begin{array}{l}\text { Weiterentwicklung der Realschulen; } \\
\text { Schulgesetz } § 7\end{array}$ & $\begin{array}{l}\text { Realschulen erhalten mehr Mittel um } \\
\text { individuelle Förderung umzusetzen. } \\
\text { Sie bieten den Hauptschulabschluss } \\
\text { und den Realschulabschluss an. } \\
\text { Klasse } 5 \text { und } 6 \text { werden als Orien- } \\
\text { tierungsstufe mit halbjährlicher } \\
\text { Wechselmöglichkeit zwischen den } \\
\text { Lernniveaus und leistungsheteroge- } \\
\text { nen Lerngruppen gestaltet }\end{array}$ \\
\hline 23.03.2016 & Bildungspläne 2016 & $\begin{array}{l}\text { Die kompetenzorientierten Bildungs- } \\
\text { pläne sind anhand von Niveaustufen } \\
\text { (statt Schularten) organisiert um } \\
\text { individualisiertes Lernen zu erleich- } \\
\text { tern. Die Leitperspektiven sind } \\
\text { unterrichtsübergreifend umzusetzen }\end{array}$ \\
\hline
\end{tabular}

Landesregierung an. Den Grünen war dabei eine schulartübergreifende, abschlussbezogene Ausgestaltung der Bildungspläne sowie die Einführung von Leitperspektiven ${ }^{4}$, die sich als „roter Faden“ durch alle Fächer ziehen, besonders wichtig, und die SPD stand ihnen dabei nicht im Weg. An dem Leitprinzip „Bildung für Toleranz und Akzeptanz von Vielfalt“ jedoch entwickelte sich ein erbitterter Streit, der über Landesgrenzen hinweg wirkte. Konservative, rechtspopulistische und pietistische Kräfte im Land warfen der Landesregierung vor, diese Leitperspektive sei eine ,pädagogische, moralische und ideologische

\footnotetext{
${ }^{4}$ Die Leitperspektiven sind: „Bildung für nachhaltige Entwicklung“, „Bildung für Toleranz und Akzeptanz von Vielfalt“, „Prävention und Gesundheitsförderung“, „Berufliche Orientierung“, „Medienbildung“" und „Verbraucherbildung“.
} 
Umerziehung“ (openpetition o. J.) und setzten sich mit einer Petition und mehreren Demonstrationen gegen ihre Umsetzung ein. Unterstützer der Bildungsplanreform organisierten Gegendemonstrationen, sodass die Polizei die Demonstranten trennen musste (z. B. Bilger 2014; Maier 2015). Nach ausführlicher Erprobung der neuen Bildungspläne und der Fortbildung von Lehrkräften gab Kultusminister Stoch die Pläne im März 2016 trotz des nach wie vor nicht beigelegten Streits als letzte Amtshandlung per Unterschrift frei (Osel 2016). Sie gelten somit ab dem Schuljahr 2016/2017 für alle allgemeinbildenden Schulen.

Ein weiterer sehr umstrittener Punkt war die Ankündigung Kretschmanns, angesichts sinkender Schülerzahlen mit einer Kürzung von 11.600 Lehrerstellen bis $2020 \mathrm{zu}$ einem ausgeglichenen Landeshaushalt beizutragen (otr und dpa 2012). Aufgrund starker Proteste der Lehrergewerkschaften, einer neuen Berechnung der Bevölkerungsprognose sowie der Aufnahme von Geflüchteten in die baden-württembergischen Schulen wurde diese Aussage im Verlauf der Regierungsperiode mehrmals revidiert und auf 1200 gesenkt (red und dpa 2016). Ein Blick auf die Haushaltspläne der Landesregierung zeigt, dass im Verlauf der Regierungsperiode keine Personalstellen gestrichen wurden. Stattdessen lässt sich das Gegenteil feststellen: Insgesamt wurden im Haushaltsplan für 2016 für Primar- und Sekundarstufe zusammen über 5700 Personalstellen mehr eingeplant als im Haushaltsplan für 2011. Deutlichen Kürzungen von knapp 2000 Personalstellen im Bereich der Grund-, Haupt- und Werkrealschulen steht ein signifikanter Zuwachs von fast 6000 Personalstellen an Gemeinschaftsschulen entgegen. Zudem wurden für Sonderschulen im Nachtragshaushalt 2016 rund $330 \mathrm{mehr}$ Personalstellen als 2011 eingeplant, was sich auf die Umsetzung der Reform zur Inklusion zurückführen lässt.

Im Folgenden wollen wir anhand einer Darstellung dreier wichtiger Reformprozesse analysieren, ob der Regierungswechsel von Schwarz-Gelb zu Grün-Rot tatsächlich - wie wir anhand der Parteiendifferenzthese erwarten würden - zu einer grundlegend anderen Bildungspolitik geführt hat. Gleichzeitig untersuchen wir, ob Pfadabhängigkeiten und Feedback-Effekte den Handlungsspielraum der grün-roten Regierungskoalition eingeschränkt oder erweitert haben. Konkret befassen wir uns im Detail mit der Abschaffung der Verbindlichkeit der Grundschulempfehlung, mit der Einführung der Gemeinschaftsschule im Zusammenhang mit der regionalen Schulentwicklung und mit der Verankerung von Inklusion im Schulgesetz. 


\section{$5 \quad$ Fallstudien}

Wir bestimmen die Präferenzen unterschiedlicher involvierter Akteure anhand von Wahlprogrammen, Positionspapieren und Pressemitteilungen. Wir betrachten sowohl Parteien als auch Verbände der Lehrer-, Eltern- und Schülerschaft. Zusätzlich greifen wir auf Informationen zurück, die wir im Rahmen von Experteninterviews mit diesen Akteuren im Zeitraum Juli/August 2015 gewonnen haben (Busemeyer und Haastert 2015). Nach einer Übersicht über den Status Quo zum Zeitpunkt der Landtagswahl 2011 beschreiben wir die Vorstellungen der Akteure und ihre Rolle im Reformprozess, um mit der aktuellen Positionierung zu enden. Wir dokumentieren somit die politischen Positionen der relevanten Akteure, wie sich diese unter Umständen geändert haben und welche Akteure sich in den Reformprozessen durchsetzen konnten. Im Anschluss erfolgt eine Einschätzung über die Reichweite der beschlossenen Reforminhalte, um die Frage zu beantworten, ob bzw. inwiefern die baden-württembergische Bildungslandschaft durch die Reform grundlegend transformiert wurde.

\subsection{Abschaffung der Verbindlichkeit der Grundschulempfehlung}

In Baden-Württemberg wurde der Übergang eines Kindes auf eine weiterführende Schule bis zum Jahr 2011 durch die verbindliche Grundschulempfehlung geregelt, die von den Grundschulen im Verlauf der vierten Klasse an die Eltern übermittelt wurde. Sofern die Eltern nicht einverstanden waren, konnte ein Test- und Beratungsverfahren eingeleitet werden, um die Grundschulempfehlung zu überprüfen (Kultusministerium 2011a). Aus Sicht des damals CDU-geführten Kultusministeriums gab es keinen Grund, diese Regelung zu verändern, da sie ein bewährtes Instrument zur Steuerung der Schülerströme im gegliederten Schulsystem war (ebenso: Exp15, Exp17, Exp4). Zudem würden Schüler*innen aus benachteiligten Familien ohne die verbindliche Grundschulempfehlung weniger häufig ein Gymnasium besuchen, weil ihre Eltern diese Schulart nicht ernsthaft für ihre Kinder in Betracht ziehen würden (CDU 2011b; Kultusministerium 2011a).

Der baden-württembergische Landeselternbeirat (LEB) hingegen forderte bereits seit Jahren die Umwandlung der verbindlichen Grundschulempfehlung in eine „echte“ Empfehlung mit ausführlicher Elternberatung im Verlauf der Grundschule und Elternwahlrecht in Bezug auf die weiterführende Schulart (LEB 2011). Auch SPD und Grüne sprachen sich in ihren Wahlprogrammen 2011 gegen die Verbindlichkeit der Grundschulempfehlung aus, da diese „,unzeitgemäß“ (SPD 2011, S. 17) 
sei und die Effekte der sozialen Herkunft auf die Bildungswege der Kinder verstärken würde (Die Grünen 2011a, S. 99). Zudem setze die „Jagd“ (VBE 2012) nach der gewünschten Empfehlung nicht nur die Kinder unter hohen Leistungsdruck, sondern auch die Lehrkräfte unter Rechtfertigungsdruck (VBE 2012, Exp3, Exp24), wie SPD und Grüne in Übereinstimmung mit der Gewerkschaft Erziehung und Wissenschaft (GEW) und dem Verband Bildung und Erziehung (VBE) - den schulartübergreifenden gewerkschaftlichen Vertretungen der Lehrkräfte - argumentierten. Aufgrund dieser negativen Effekte der verbindlichen Grundschulempfehlung versprach die grün-rote Koalition die Einführung einer qualifizierten Elternberatung während der Grundschulzeit und das Elternwahlrecht (Die Grünen und SPD 2011, S. 6).

Direkt nach Amtsübernahme erarbeitete das Kultusministerium unter Anhörung verschiedener Akteure eine Gesetzesänderung zur Abschaffung der Verbindlichkeit der Grundschulempfehlung noch im Schuljahr 2011/2012. Im Verlauf dieses Prozesses wies der Landesschülerbeirat darauf hin, dass eine Umstellung zwar zu begrüßen, aber im laufenden Schuljahr noch zu früh sei, da eine ausreichende Elternberatung dann nicht möglich sei (Kultusministerium 2011b). Aus Sicht der Opposition war die schnelle Initiative zur Abschaffung der Verbindlichkeit nicht nur überhastet, sondern auch unnötig, da es bereits eine Elternberatung und Revisionsmöglichkeiten gebe (CDU 2011b). Zudem, so mutmaßte die Opposition, bringe die Reform keine Entlastung von Kindern und Lehrern in Grundschulen, sondern sei vielmehr eine Maßnahme, um das gegliederte Schulsystem zugunsten der Gemeinschaftsschule anzugreifen (Allgöwer 2011).

Trotz dieser Widerstände beschloss der Landtag am 17. Dezember 2011 die Abschaffung der Verbindlichkeit der Grundschulempfehlung. Seit dem Schuljahr 2011/2012 treffen demnach die Erziehungsberechtigten von Viertklässler*innen selbst die Entscheidung über eine weiterführende Schulart für ihr Kind und werden dabei von den Grundschullehrkräften durch kontinuierliche Beratungsgespräche unterstützt. Die weiterführende aufnehmende Schule hat kein Recht darauf, die Grundschulempfehlung einzusehen.

Infolge der Gesetzesänderung stiegen die Übergangsquoten an Realschulen und Gymnasien in den Schuljahren 2012/2013 und 2013/2014 stärker an als zuvor; dieser Trend ist seit dem Schuljahr 2013/2014 wieder leicht zurückgegangen (Landesinstitut für Schulentwicklung 2015, S. 94). ${ }^{5}$ Durch die veränderten

\footnotetext{
${ }^{5}$ So sank die Übergangsquote auf Werkrealschulen von 23,7 \% im Schuljahr 2011/2012 auf 9,3\% im Schuljahr 2014/2015, während die Übergangsquote auf Gymnasien von 40,9\% im Schuljahr 2011/2012 auf 44,6 \% im Schuljahr 2013/2014 stieg und Realschulen eine Erhöhung von 34,2 \% im Schuljahr 2011/2012 auf 37,1 \% im Schuljahr 2012/2013 verzeichneten (Landesinstitut für Schulentwicklung 2015, S. 94).
} 
Möglichkeiten der Schulwahl erhöhte sich offiziellen Statistiken zufolge insbesondere an Realschulen die Heterogenität der Schülerschaft in Bezug auf ihre Grundschulempfehlungen und es kam zu einer - zumindest temporären - Erhöhung der Nichtversetzen-Quote (Landesinstitut für Schulentwicklung 2015, S. 101, 131). Diese Entwicklungen zogen große mediale Aufmerksamkeit auf sich und auch die Gegner der Gesetzesänderung wiesen Jahr um Jahr auf die negativen Effekte von Nichtversetzung oder Schulwechsel auf die Lernmotivation und das Selbstbewusstsein der Kinder hin (Allgöwer 2014; Breining 2015; Kultusministerium 2013c; PHV 2013, 2014).

Für die Legislaturperiode 2016-2021 sieht jedoch keine der Parteien in ihren Wahlprogrammen eine direkte Wiedereinführung der Verbindlichkeit der Grundschulempfehlung vor: Grüne und SPD halten die unverbindliche Grundschulempfehlung in ihrer derzeitigen Form für richtig und wollen den Dialog zwischen Eltern und Grundschule weiter fördern (SPD 2016, S. 25 f.; Die Grünen 2016, S. 95). Die CDU verspricht, ein hochwertiges Beratungskonzept ab Klasse $1 \mathrm{zu}$ etablieren, um die Grundschulempfehlung überflüssig zu machen und sieht für Real- und Werkrealschüler*innen eine weitere Bildungswegeempfehlung nach Klasse 6 vor (CDU 2016, S. 26). Die FDP setzt sich dahingegen für eine bessere Personalausstattung an Grundschulen für die Elternberatung ein und verspricht aufnehmenden Schulen das Recht, sich über die Grundschulempfehlung ihrer Schüler*innen informieren zu können. Sollten sich aber durch diese Maßnahmen die Nichtversetzen-Quoten nicht bis 2017 verringern, erwägt die FDP als einzige größere Partei eine Wiedereinführung der Verbindlichkeit der Grundschulempfehlung (FDP 2016, S. 20-25).

Insgesamt zeigt sich, dass trotz der harschen Kritik vonseiten der Opposition auch CDU und FDP auf dem neuen Status quo aufbauen wollen. Es scheint, dass die Einführung des Elternwahlrechts bei einem Großteil der Betroffenen auf Unterstützung stößt und somit mit hoher Wahrscheinlichkeit langfristig erhalten bleiben wird.

\subsection{Gemeinschaftsschule und regionale Schulentwicklung}

Im Jahr 2011 bestand das gegliederte Schulsystem in Baden-Württemberg aus Hauptschulen, Werkrealschulen, Realschulen, Gymnasien und Förderschulen. Aus Sicht von CDU und FDP, des Philologenverbandes und der beruflichen Schulen entsprach dieses differenzierte Schulsystem dem Motto „Kein Abschluss ohne Anschluss“ und sei daher bestens dazu geeignet jede*n Schüler*in entsprechende 
seiner/ihrer Begabungen zu fördern (CDU 2011a, S. 40 f.; BLV 2014; Exp4, Exp17; FDP 2010, S. 12; FDP 2011, S. 37). Dieser Überzeugung entsprechend wurde Anträgen von Schulträgern, integrative Schulen einzurichten, vom CDUgeführten Kultusministerium nicht stattgegeben. Auch der Vorschlag der FDP, Verbünde verschiedener Schularten und individuelle Lernformen zu fördern (FDP 2010, S. 8, 12) wurde nicht umgesetzt.

Angesichts dieser Politik warfen die Grünen und die SPD der ehemaligen Landesregierung eine „,ideologischen Fixierung“ auf das dreigliedrige System (SPD 2008) und eine „Blockadepolitik“ gegenüber Schulinnovationen (Die Grünen 2011a, S. 100) vor. Zudem sahen SPD und Grüne ebenso wie der Städtetag und der Landeselternbeirat eine große Herausforderung für das gegliederte Bildungssystem in den sinkenden Schülerzahlen, aufgrund derer die Zukunft vieler Schulstandorte im ländlichen Raum bedroht war (Die Grünen 2011a, S. 103, 107; Die Grünen 2011b; Exp21; Exp18; Exp11; Städtetag 2011).

Die Verankerung der Gemeinschaftsschule im Schulgesetz war daher eine Priorität der grün-roten Landesregierung. Integrative und inklusive, 10-jährige Gemeinschaftsschulen sollten das dreigliedrige System aufbrechen und mit neuen Lehr- und Lernformen längeres gemeinsames Lernen ermöglichen - was ein Beitrag für mehr Bildungsgerechtigkeit sei. Das Angebot aller Niveaustufen fördere zudem den Erhalt wohnortnaher Schulstandorte im Kontext sinkender Schülerzahlen, und die Einführung des Schulganztags verbessere die Vereinbarkeit von Familie und Beruf (Die Grünen und SPD 2011, S. 6).

Viele Akteure äußerten sich positiv oder zumindest abwartend gegenüber der geplanten Gemeinschaftsschule. So begrüßten der Landeseltern- und der Landesschülerbeirat die Einführung der Gemeinschaftsschule aus pädagogischen und sozialen Gründen (Exp18; LEB 2012; Kultusministerium 24.10.2011a), der Städtetag aus schulstrukturellen Überlegungen (Städtetag 2012), und Wirtschaftsvertreter aufgrund der Hoffnung auf bessere soziale Fähigkeiten und Reife der Absolventen (Exp16, Exp8). Jedoch kritisierten viele dieser prinzipiellen Reformbefürworter sehr, dass die Gemeinschaftsschule eingeführt werden sollte, ohne zuvor ein Gesamtkonzept für die Schullandschaft und die Finanzierung entwickelt zu haben (Gemeindetag 2012, 2013a; Städtetag 2012; Exp18). Ein großer Kritikpunkt war auch die Bevorzugung der Gemeinschaftsschulen im Hinblick auf Personalversorgung, bauliche Maßnahmen und finanzielle Ausstattung. Dies sei nicht im Sinne einer gerechten Ressourcenverteilung und führe zu Fehlanreizen für die Einrichtung einer Gemeinschaftsschule (LEB 2012; Gemeindetag 2012; Städtetag 2012).

Der vorgesehenen Ressourcenverteilung zufolge blieben Neid und Existenzängste unter den Schularten nicht aus. Die ,ideologisch gesteuerte 
Bildungspolitik“ (RLV 2013) der grün-roten Landesregierung habe das Ziel, die Gemeinschaftsschule auf Kosten der bestehenden, leistungsfähigen Schularten durchzusetzen (CDU 2013c; Exp 4; Exp 17; Exp23; PHV 2012; RLV 2012). Neben den Lehrerverbänden der Gymnasial-, Realschul- und Berufsschullehrer warfen auch CDU und FDP der Landesregierung vor, dass die Gemeinschaftsschule das gegliederte Schulsystem zugunsten der „Einheitsschule“ untergraben solle und sie damit die Leistungsfähigkeit des baden-württembergischen Bildungssystems zu zerstören drohe (o. A. 2011; FDP 2013, S. 4; CDU 2013c, S. 1 f.).

Trotz dieser massiven Kritik und zahlreicher Änderungsvorschläge veränderte das Kultusministerium weder die Reihenfolge der vorgesehenen Reformen, noch die Ausstattung der Gemeinschaftsschulen, da, so die Landesregierung, die Umstellung auf die neue Schulform einen Mehraufwand rechtfertige (Exp21, Exp22). Am 21. April 2012 fügte daher der Landtag dem Schulgesetz den Paragrafen $\S 8 \mathrm{a}$ hinzu, in dem die Gemeinschaftsschule gesetzlich festgeschrieben und ihre Ausgestaltung geregelt ist.

Im Schuljahr 2012/2013 begannen 41 Schulen mit über 2000 Schüler*innen als Gemeinschaftsschulen, im Schuljahr 2016/2017 gibt es bereits knapp 300 Gemeinschaftsschulen mit deutlich über 20.000 Schüler*innen (Landesinstitut für Schulentwicklung 2015, S. 119-121; Kultusministerium 2016). Davon sind die meisten ehemalige Werkrealschulen, aber auch über 30 ehemalige Realschulen oder Gymnasien gehören zu den Gemeinschaftsschulen (ibid.; Schwarz-Jung 2014). Die Resonanz von Schüler*innen, Eltern und Lehrkräften an den Gemeinschaftsschulen ist überwiegend positiv (Busemeyer und Haastert 2015), doch es werden auch Schwachstellen identifiziert. So sei die Arbeitsbelastung der Lehrkräfte durch die verbale und schriftliche Leistungsbewertung deutlich höher, und einige Standorte hätten sich nicht aus Überzeugung, sondern aus Standortsicherungsinteresse in eine Gemeinschaftsschule entwickelt (ibid.; PHV 2016; Exp11; Exp3).

Um die Herausforderung anzugehen, eine flächendeckende Versorgung mit Schulen und Abschlussmöglichkeiten auch in ländlichen Regionen BadenWürttembergs zu erhalten (und möglicherweise als Antwort auf den vielfach geäußerten Bedarf nach einer Schulentwicklungsplanung), erarbeitete das Kultusministerium im Verlauf des Jahres 2013 einen Gesetzesentwurf zur regionalen Schulentwicklung. Dieses Gesetzesvorhaben wurde deutlich weniger kontrovers diskutiert als die Einführung der Gemeinschaftsschule. Ein Grund hierfür war, dass sowohl das Ziel - vielfältige Abschlussmöglichkeiten und die zumutbare Erreichbarkeit der Schulstandorte zu erhalten - als auch das Verfahren - Beteiligungs- und Dialogverfahren vor Ort einzurichten - prinzipiell von vielen Seiten 
geteilt wurde (CDU 2013b; Städtetag 2013; Gemeindetag 2013b; Exp18). Kritik bezog sich hauptsächlich auf die Nennung der Verfahrensbeteiligten im Gesetz (LEB 2014; Exp18; CDU 2013b; Exp7) sowie die Festlegung von Mindestschülerzahlen (CDU 2013b; Exp20; Exp12).

Am 22. Mai 2014 beschloss der Landtag die vom Kultusministerium erarbeitete Änderung des Schulgesetzes zur regionalen Schulentwicklung für weiterführende Schulen. Demnach wird ein Verfahren der regionalen Schulentwicklung eingeleitet, wenn eine im Gesetz vorgegebene Mindestschülerzahl unterschritten wird oder wenn es von einem Schulträger, einer Gemeinde oder einem Landkreis gewünscht wird. Alle „von der schulorganisatorischen Maßnahme Berührten“ (Schulgesetz § 30c Absatz 2) sind an den Dialogverfahren über die Schulentwicklungsplanung zu beteiligen, insbesondere weitere Schulen und Gemeinden im relevanten Gebiet. Dies verlangt eine große Kooperations- und Kompromissbereitschaft von den Verfahrensbeteiligten. Wird kein Konsens erreicht, kommt es zu einem Schlichtungsverfahren durch die obere Schulaufsichtsbehörde. Laut der SPD wurden seit der gesetzlichen Regelung der regionalen Schulentwicklung $93 \%$ der 125 bisher abgeschlossenen Verfahren konsensual gelöst (SPD 2015).

Der mehrheitlich positiven Auffassung gegenüber der regionalen Schulentwicklung zufolge sehen alle Parteien in ihren Wahlprogrammen für 2016 von einer grundlegenden Veränderung dieser Regelung ab (Bündnis90/Die Grünen 2016, S. 95; SPD 2016, S. 26). Einzig die FDP plant, Bildungsregionen einzurichten und Inklusion gesetzlich in die regionale Schulentwicklung zu integrieren (FDP 2016, S. 25).

Deutlich größere Differenzen zwischen den Parteien bestehen jedoch hinsichtlich der Zukunft der Gemeinschaftsschulen und des Schulsystems allgemein: CDU und FDP wollen das differenzierte Bildungssystem erhalten und Gemeinschaftsschulen nicht weiter genehmigen oder bevorzugt finanzieren. Um Schulen im ländlichen Raum zu erhalten, sehen sie Verbundschulen vor, also die organisatorische Zusammenlegung von Werkreal- und Realschulen unter einer gemeinsamen Schulleitung. Laut CDU soll eine solche „Realschule Baden-Württemberg“ die 5. und 6. Klasse in Form einer Orientierungsstufe mit leistungsheterogenen Schülergruppen organisieren und den halbjährlichen Wechsel des Lernniveaus zulassen. Ab Klasse 7 soll (in den Kernfächern) leistungsdifferenzierter Unterricht stattfinden (CDU 2016, S. 27, 29). Die FDP dagegen möchte den Schulen selbst die Wahl der Schulform sowie der pädagogisch-inhaltlichen Ausrichtung überlassen (FDP 2016, S. 25).

Grüne und SPD dagegen streben ein Zwei-Säulen-Modell an, das aus dem allgemeinbildenden Gymnasium einerseits und einer integrativen Säule andererseits bestehen soll. Die integrative Säule soll sowohl aus Gemeinschaftsschulen als 
auch aus den weiterentwickelten Realschulen, Haupt- und Werkrealschulen bestehen. SPD und Grüne sehen vor, Realschulen durch Fortbildungen und Lehrerstundenzuweisung bei der Umsetzung von individualisiertem Lernen zu unterstützen; auf Wunsch soll ihnen auch eine Umwandlung in Gemeinschaftsschulen möglich sein. An der Ausstattung von Gemeinschaftsschulen sowie der Einrichtung der gymnasialen Oberstufe an Gemeinschaftsschulen halten die Parteien fest (Die Grünen 2016, S. 99 f.; SPD 2016, S. 26 f.).

Anhand der Wahlprogramme der Parteien lässt sich feststellen, dass die Einführung der Gemeinschaftsschule, die regionale Schulentwicklung und die Weiterentwicklung der Realschulen, die im Dezember 2015 beschlossen wurde, einen strukturellen Wandel im Schulsystem initiiert hat: Sowohl die Gemeinschaftsschule als auch die weiterentwickelte Realschule bieten verschiedene Abschlussarten an und setzen individualisierte Lernformen ein. Viele Akteure, vom Landeselternbeirat über den Städtetag bis hin zu Wirtschaftsvertretern, stehen dieser Entwicklung positiv gegenüber, während Realschul-, Gymnasial- und Berufsschullehrer sowie die Oppositionsparteien sie kritisieren. Dennoch plant keine der vier größten Parteien in Baden-Württemberg ein „Zurück“ ins Schulsystem des Jahres 2011. Es erscheint daher wahrscheinlich, dass sich auf längere Frist auch in Baden-Württemberg ein zweigliedriges Schulsystem etablieren wird - ob die Gemeinschaftsschule in ihrer aktuellen Form bestehen bleibt, hängt jedoch von dem Wahlergebnis ab.

\subsubsection{Inklusion}

Die Umsetzung von Inklusion war in Baden-Württemberg zum Zeitpunkt der Amtsübernahme durch die grün-rote Landesregierung bereits im Rahmen von Schulversuchen auf den Weg gebracht. Die CDU-geführte Vorgängerregierung hatte im Jahr 2010 Modellregionen - Stuttgart, Mannheim, Konstanz, Biberach und Freiburg - eingerichtet, in denen Formen inklusiven Unterrichts erprobt wurden und sah vor, die dort gesammelten Erfahrungen in eine Gesetzesänderung zum Schuljahr 2013/2014 einfließen zu lassen (Rodenhausen 2010).

Wie bereits angedeutet, setzten sich im Koalitionsvertrag zwischen den Grünen und der SPD weitgehend die Vorstellungen der Grünen durch. So versprach die neue Landesregierung, den Anspruch auf sonderpädagogische Förderung in allgemeinen Schulen gesetzlich zu verankern, das Elternwahlrecht einzuführen, das Zwei-Pädagogen-Prinzip in inklusivem Unterricht zu realisieren und Sonderschulen für Kinder ohne Behinderung zu öffnen (Die Grünen und SPD 2011, S. 7). Nach der Wahl wurden jedoch zunächst andere Reformen angegangen. Eine interfraktionelle Arbeitsgruppe, wie sie die ehemalige Kultusministerin Warminski-Leitheußer zu Beginn ihrer Amtszeit vorgeschlagen hatte (und welche die 
FDP mehrmals einforderte) richtete das Kultusministerium nicht ein (FDP 2012, 2014b). Dementsprechend warf die Opposition dem SPD-geführten Kultusministerium Desinteresse und sogar „unterlassene Hilfeleistung“ (FDP 2013) vor (auch: CDU 2013a) und erarbeitete indes eigene Eckpunktepapiere zu Inklusion (CDU 2014; FDP 2014a).

Erst im Februar 2015 legte Grün-Rot einen Gesetzesentwurf zur Umsetzung des Inklusionsprinzips vor - und damit deutlich später als von der ehemaligen CDU-FDP-Koalition ebenso wie von Grün-Rot selbst geplant (Kultusministerium 2012, 2015; CDU 2014). Dieser nahm weitgehend die Vorschläge und Forderungen vorheriger Eckpunktepapiere von CDU und FDP auf (FDP 2014a; CDU 2014, 2015). Die Kritikpunkte der Opposition bezogen sich lediglich auf die Personalallokation und die Lehrerfortbildung (CDU 2015; Exp18; Exp19), während Städte- und Gemeindetag bemängelten, dass das beschränkte Elternwahlrecht zu unklar definiert sei und dass die geplanten gruppenbezogenen Lösungen - mehrere Kinder mit Behinderung werden an einer Schule unterrichtet - organisatorisch und finanziell zu aufwendig seien. Die Einrichtung von Schwerpunktschulen bestimmte Schulen nehmen Kinder mit ähnlichen Behinderungen auf - sei für eine Übergangszeit besser geeignet (Gemeindetag 2015; Städtetag 2015).

Die am 15. Juli 2015 im Landtag beschlossene Änderung des Schulgesetzes zur Umsetzung von Inklusion an allgemeinen Schulen entspricht weitgehend dem Gesetzentwurf: Die Sonderschulpflicht ist abgeschafft, und alle Sonderschulen- und Förderschulen werden zu Sonderpädagogischen Bildungs- und Beratungszentren (SBBZ) mit Angeboten für Kinder mit und ohne Behinderung weiterentwickelt, sodass die Bildung und Erziehung von Schüler*innen mit Behinderung eine Aufgabe aller Schulen wird. Die Landesregierung sieht Bildungswegekonferenzen vor, in deren Rahmen Eltern über die Fördermöglichkeiten für ihr Kind an allgemeinen Schulen und SBBZ beraten werden. Die Entscheidung über den geeigneten Lernort treffen die Eltern, die Wahl einer konkreten Schule ist jedoch nicht möglich. Weiterhin wird inklusiver Unterricht durch gruppenbezogene Lösungen umgesetzt und das Zwei-Pädagogen-Prinzip angestrebt (Kultusministerium 2015b). Insgesamt lassen sich bei der Umsetzung der Inklusion also durchaus Pfadabhängigkeiten feststellen. Innerhalb der SBBZ bleibt das Angebot spezialisierter Bildung für behinderte Kinder - also das Sonder- und Förderschulangebot - erhalten, obwohl der Erhalt der Sonderschulen im Verlauf des Reformprozesses von Grün-Rot stark infrage gestellt worden war.

Für die Legislaturperiode 2016-2021 sehen die Parteien in Bezug auf die weitere Förderung von Inklusion verschiedene Maßnahmen, aber keine grundlegenden Änderungen vor. Die CDU möchte Eltern von Kindern mit Behinderung einen Elternlotsen sowie einen Landesombudsmann zur Seite zu stellen, 
Sonderschulen primär mit Sonderpädagogen zu versorgen und freien Schulen mehr Unterstützung geben (CDU 2016, S. 32). Die FDP will den Sonderschulstatus gesetzlich festschreiben, freie Schulen besser unterstützen, und den Finanzierungsmechanismus verändern (FDP 2016, S. 26 f.). Die Grünen sehen vor, zusätzliche Lehrerstellen und mehr finanzielle Mittel für die Umsetzung von Inklusion bereitzustellen (Die Grünen 2016, S. 106 f.). Die SPD schließlich setzt den Fokus auf die weitere Aus- und Fortbildung der Lehrkräfte (SPD 2016, S. 29). Es besteht daher kein Zweifel daran, dass das qualifizierte Elternwahlrecht bestehen bleibt und sonderpädagogische Förderung zukünftig sowohl an den SBBZ als auch an allgemeinbildenden Schulen angeboten wird.

\section{Analyse und abschließende Bewertung: Inkrementeller Wandel oder Systemwechsel?}

Die Fallstudien belegen im Bereich der Bildungspolitik eine hohe Reformdichte. Innerhalb eines vergleichsweise kurzen Zeitraums wurde eine Vielzahl an Reformen angegangen und auch gegen Widerstände durchgesetzt. Damit konnten SPD und Grüne die von ihnen bereits länger geforderte Schulstrukturreform hin zu einem zweigliedrigen System angehen. Für den Bereich der Schulstrukturreformen lässt sich daher festhalten, dass die positiven Feedback-Effekte (Beharrungskräfte für das dreigliedrige Schulsystem) überwunden werden konnten. Dies mag zu einem großen Teil an den starken negativen Feedback-Effekten gelegen haben, die wir in Abschn. 3 beschrieben haben. Hierbei ist allerdings zu berücksichtigen, dass die Vorschläge der Grün-roten Regierung zu Strukturreform in gewissem Sinne bereits die Wirkmächtigkeit von Pfadabhängigkeiten widerspiegeln: Im Unterschied zu den Reformdiskussionen der 1970er Jahre stand nicht mehr die vollständige Abschaffung des dreigliedrigen Systems zugunsten eines integrierten Gesamtschulsystems auf der Agenda, sondern lediglich die Einführung eines zweigliedrigen Schulsystems mit einer integrativen ersten Säule und dem weiterhin zu erhaltenden Gymnasium als zweite Säule.

Dennoch werden viele Reformen auch über einen Regierungswechsel hinaus Bestand haben, wie der kurze Ausblick auf die Positionen der Parteien im Wahlkampf 2016 gezeigt hat. Dies sind insbesondere die Abschaffung der Verbindlichkeit der Grundschulempfehlung, die Umsetzung des Inklusionsprinzips (hierbei insbesondere die Einführung des Elternwahlrechts) und die Reformen der regionalen Schulplanung. Aber auch die Gemeinschaftsschule - also dasjenige Reformprojekt, das die meisten Kontroversen auf politischer Ebene auslöste - wird mit hoher Wahrscheinlichkeit als Schulform auch unter einer bürgerlichen 
Regierung weiter bestehen bleiben, denn die Vorschläge der gegenwärtigen Oppositionsparteien zielen eher auf eine Konsolidierung des Bestehenden statt auf den Rückbau der Gemeinschaftsschulen. Insofern kann durchaus konstatiert werden, dass die grün-roten Reformen einen langfristigen Systemwandel von einem eher "traditionellen“ dreigliedrigen System hin zu einem Modell mit „modernisierten Strukturen“ (Helbig und Nikolai 2015, S. 261) eingeleitet hat. Dieser Systemwandel wird jedoch ein inkrementeller Prozess sein und kein radikaler Wandel (vgl. Streeck und Thelen 2005 zur Theorie von inkrementellem transformativem Wandel). Das dabei zu beobachtende Muster inkrementellen Wandels kann als eine Kombination aus „institutional layering“ und „conversion" beschrieben werden, denn die grün-rote Regierung setzte mit der Gemeinschaftsschule einen neuen Schultypus neben die bereits bestehenden. Sie tat dies allerdings, indem bestehende Institutionen (konkret: Schulen) in Gemeinschaftsschulen umgewandelt wurden. Langfristig könnte dies zu einem Prozess des ,institutional displacement" führen, denn die neuen Gemeinschaftsschulen könnten bei fortgesetzter, teils selektiver Förderung die bestehenden traditionellen Schulformen ersetzen.

Die grün-rote Regierung verfolgte bei den Bildungsreformen einen bestimmten politischen Ansatz, der sicherlich zu diesem relativen Erfolg der Reformpolitik beigetragen hat. Wie kann dieser Ansatz charakterisiert werden? Zunächst ist auffällig, dass die grün-rote Regierung nicht wie in einigen anderen Bildungsländern bereits zu Anfang ihrer Regierungszeit den Schulterschluss mit den Oppositionsparteien gesucht hat, um im Rahmen eines „Schulkompromisses“ oder eines „Bildungsfriedens“ Strukturreformen auszuhandeln, die auch langfristig über Regierungswechsel hinweg Bestand haben. Stattdessen hat die grün-rote Regierung ein ambitiöses und von den Oppositionsparteien dementsprechend hart kritisiertes Reformprogramm eingeleitet und in Gesetzesänderungen umgesetzt. Bei vielen Reformprojekten - insbesondere bei der Einführung der Gemeinschaftsschule - stand eine Reformkoalition angeführt von den grün-roten Regierungsparteien, unterstützt von Eltern- und einigen Lehrerverbänden und -gewerkschaften, die den Regierungsparteien politisch nahestehen, gegen die Oppositionsparteien und andere Lehrerverbände, die das traditionelle System verteidigten (insbesondere der Philologenverband sowie die Verbände der Realschul- und Berufsschullehrer).

Die harte Linie gegenüber der Opposition wurde allerdings dadurch relativiert, dass die Reformen in wichtigen Punkten die Rechte der Eltern stärkten (wie etwa bei der Abschaffung der Verbindlichkeit der Grundschulempfehlung oder dem Elternwahlrecht bei der Inklusion) und genug Spielraum für lokale Initiativen und Beteiligung ließen (wie bei der Einführung der Gemeinschaftsschule und der 
regionalen Schulentwicklung). Eine Alternative wäre beispielsweise gewesen, im Rahmen eines „top-down“-Ansatzes Gemeinschaftsschulen per Regierungsbeschluss umzuwandeln oder die Standorte von Schulen im Bundesland zentral von Stuttgart aus festzulegen. Der von Grün-Rot stattdessen implementierte „bottomup“-Ansatz verbunden mit einer Politik der Stärkung der Beteiligungsrechte half sicherlich dabei, bei den entsprechenden Verbänden, Kommunalpolitikern und lokalen Stake-Holdern Unterstützung für die Reformen zu schaffen. Langfristig könnten dadurch wirkmächtige Feedback-Effekte entstehen, denn zukünftige Regierungen werden sich schwertun, die nun bestehenden Wahlmöglichkeiten und Beteiligungsrechte wieder zu beschränken.

Des Weiteren wurden im Rahmen einer "Politik des Goldenen Zügels“ beträchtliche finanzielle Anreize gesetzt, dass sich bestehende Schulen in Gemeinschaftsschulen umwandeln. Aufgrund der besseren finanziellen und personellen Ausstattung der Gemeinschaftsschulen sahen auch pragmatisch veranlagte Lokalpolitiker der Oppositionsparteien in der Einführung der Gemeinschaftsschule eine Chance, die lokalen Schullandschaften zu konsolidieren und attraktiver zu machen (vgl. Busemeyer und Haastert 2015). Durch die finanziellen Anreize konnte die Landesregierung somit zusätzliche Unterstützer für ihre Reformen gewinnen, die traditionell nicht zu ihrem Wählerkreis gehörten. Dies kann die neuen Feedback-Effekte verstärken, da Kürzungen finanzieller Mittel immer mit politischen Konflikten verbunden sind.

Trotz dieser Stärken sind jedoch auch einige Schwachpunkte offensichtlich geworden. Die hohe Reformdichte hat die Beteiligten, insbesondere die Lehrerschaft, teilweise überfordert und bei diesen für erheblichen Unmut gesorgt. Durch die Einführung der Gemeinschaftsschule und die Umsetzung des Inklusionsprinzips müssen sich Lehrer*innen mit neuen Lernmethoden und -inhalten auseinandersetzen, die in der Lehreraus- und -weiterbildung noch nicht systematisch integriert sind. Die graduelle Einführung der Gemeinschaftsschule trägt außerdem dazu bei, dass die Schulstrukturen auf lokaler Ebene zunächst heterogener und komplexer werden, was weitere Steuerungsprobleme nach sich ziehen und generell zu einer Verunsicherung der Schüler- und Elternschaft beitragen könnte.

Aus theoretischer Perspektive ist die grün-rote Bildungspolitik ein interessanter Fall, denn erst auf längere Frist wird sich zeigen, in welchem „Gleichgewicht“ sich die Schulstruktur stabilisieren wird. Während die grün-rote Landesregierung in diesem hochkontroversen Politikfeld das Angebot an Schultypen letztlich erhöht hat, wird die Nachfrage nach den verschiedenen Schularten durch Eltern und Schüler*innen darüber entscheiden, welche davon langfristig überleben werden. Einen solchen Prozess differenziellen Wachstums anzuregen scheint eine Möglichkeit zu sein, hochpolitisierte Politikbereiche zu reformieren, da dadurch auch kurzfristige Konflikte entschärft werden können. 


\section{Anhang I: Liste der Expert*innen}

\begin{tabular}{l|l|l}
\hline Experte*in & Stake-Holder & Kalenderwoche des Interviews (2015) \\
\hline Exp3 & Lehrergewerkschaft & KW 28 \\
\hline Exp4 & Lehrkräfte (Gymnasien) & KW 29 \\
\hline Exp7 & Privatschulen & KW 29 \\
\hline Exp8 & Arbeitgeber & KW 29 \\
\hline Exp11 & KommunaleVerwaltung & KW 30 \\
\hline Exp12 & Kommunale Verwaltung & KW 30 \\
\hline Exp15 & Bildungswissenschaft & KW 30 \\
\hline Exp16 & Arbeitgeber & KW 30 \\
\hline Exp17 & Lehrkräfte (Berufsschulen) & KW 30 \\
\hline Exp18 & Familien & KW 30 \\
\hline Exp19 & Lehrkräfte (Sonderschulen) & KW 31 \\
\hline Exp20 & Politiker (Opposition) & KW 31 \\
\hline Exp21 & Politiker (Regierungsfraktion) & KW 31 \\
\hline Exp22 & Politiker (Regierungsfraktion) & KW 31 \\
\hline Exp23 & Lehrer (Realschulen) & KW 32 \\
\hline Exp24 & Lehrergewerkschaft & KW 32 \\
\hline
\end{tabular}

\section{Literatur}

Allgöwer, R. 2011. Eltern bekommen das letzte Wort. Stuttgarter Zeitung, 10.11.2011. http://www.stuttgarter-zeitung.de/inhalt.grundschulempfehlung-eltern-bekommen-dasletzte-wort.0338cb1f-5a6d-4af1-b084-e8b017f51540.html. Zugegriffen: 05. Apr. 2016.

Allgöwer, R. 2014. CDU im Landtag - Sitzenbleiberzahlen nur Spitze des Eisbergs. Stuttgarter Zeitung, 15.08.2014. http://www.stuttgarter-zeitung.de/inhalt.cdu-im-landtagsitzenbleiberzahlen-nur-spitze-des-eisbergs.69acb878-7911-44f7-995e-1ac78cfdb83f. html. Zugegriffen: 05. Apr. 2016.

Ansell, B.W. 2008. University challenges: Explaining institutional change in higher education. World Politics 60:189-230.

Autorengruppe Bildungsberichterstattung. 2012. Bildung in Deutschland 2012: Ein indikatorgestützter Bericht mit einer Analyse zur kulturellen Bildung im Lebenslauf. Berlin: Kultusministerkonferenz, Bundesministerium für Bildung und Forschung, wbv.

Autorengruppe Bildungsberichterstattung. 2014. Bildung in Deutschland 2014: Ein indikatorgestützter Bericht mit einer Analyse zur Bildung von Menschen mit Behinderungen. Berlin: Kultusministerkonferenz, Bundesministerium für Bildung und Forschung, wbv. 
Bilger, C. 2014. Demo in Stuttgart - Bildungsplan-Gegner ausgebremst. http://www.stuttgarter-zeitung.de/inhalt.demo-in-stuttgart-bildungsplan-gegner-ausgebremst.df5fb98ddadd-413b-8cf7-c1c93eb64830.html. Zugegriffen: 05. Apr. 2016.

Blanck, J., B. Edelstein, und J. J. W. Powell. 2014. Auf dem Pfad zur inklusiven Bildung? Schulreformen in Deutschland und die UN-Behindertenrechtskonvention. In Inklusion und Chancengleichheit: Diversity im Spiegel von Bildung und Didaktik, Hrsg. S. SchuSener, N. Bernhardt, M. Hauser, und F. PoSe, 97-104. Bad Heilbrunn: Verlag Julius Klinkhardt.

BLV. 2014. Strategische Ziele des BLV. http://blv-bw.de/wp-content/uploads/2013/11/Strategische-Ziele-BLV-08.12.2014-1.pdf. Zugegriffen: 05. Apr. 2016.

Boix, C. 1997. Political parties and the supply side of the economy: The provision of physical and human capital in advanced economies, 1960-1990. American Journal of Political Science 41(3): 814-845.

Breining, T. 2015. Versetzungszahlen in Baden-Württemberg - In Städten wird öfter sitzengeblieben. Stuttgarter Zeitung, 23.03.2015. http://www.stuttgarter-zeitung.de/inhalt. versetzungszahlen-in-baden-wuerttemberg-in-staedten-wird-oefter-sitzengeblieben. a6a9b2e9-6c64-49b0-9362-1ddbbc61c20e.html. Zugegriffen: 05. Apr. 2016.

Busemeyer, M. R. 2007. The determinants of public education spending in 21 OECD democracies, 1980-2001. Journal of European Public Policy 14 (4): 582-610.

Busemeyer, M. R. 2009. Social democrats and the new partisan politics of public investment in education. Journal of European Public Policy 16 (1): 107-126.

Busemeyer, M. R. 2015. Skills and inequality: The political economy of education and training reforms in Western welfare states. Cambridge: Cambridge University Press.

Busemeyer, M. R., und S. Haastert. 2015. Kontrovers aber erfolgreich!? Eine Zwischenbilanz grün-roter Bildungsreformen in Baden-Württemberg. Stuttgart: Fritz-Erler-Forum der Friederich-Ebert Stiftung.

Busemeyer, M. R., und C. Trampusch. 2013. Liberalization by exhaustion: Transformative change in the German welfare state and vocational training system. Zeitschrift für Sozialreform 59 (3): 291-312.

Busemeyer, M. R., S. Franzmann, und J. Garritzmann. 2013. Who owns education? Cleavage structures in the partisan composition over educational expansion. West European Politics 36 (3): 521-546.

Castles, F. G. 1982. The impact of parties on public expenditure. In The impact of parties: Politics and policies in democratic capitalist states, Hrsg. F. G. Castles, 21-96. London: Sage.

CDU. 2011a. In der Tat besser. Regierungsprogramm der CDU-Baden-Württemberg 20062011. Stuttgart: CDU.

CDU. 2011b. Zweite Beratung des Gesetzentwurfs der Landesregierung - Gesetz zur Änderung des Schulgesetzes für Baden-Württemberg. Rede von Georg Wacker MdL. http:// fraktion.cdu-bw.de/fileadmin/user_upload/infothek/Bildung/2011-12-08_Wacker_zu_ Abschaffung_Grundschulempfehlung.pdf. Zugegriffen: 05. Apr. 2016.

CDU. 2013a. Unsere Position zum Thema Inklusion auf dem Weg zu einem inklusiven Bildungssystem. Beschluss des CDU-Landesvorstandes. http://www.cdu-bw.de/uploads/ media/2013-01-12-Papier-Inklusion.pdf. Zugegriffen: 05. Apr. 2016. 
CDU. 2013b. Meldungen: So wird die regionale Schulentwicklung zu einem reinen Schulschließungsprogramm! http://fraktion.cdu-bw.de/meldung/artikel/so-wird-die-regionaleschulentwicklung-zu-einem-reinen-schulschliessungsprogramm.html. Zugegriffen: 05. Apr. 2016.

CDU. 2013c. Leistungsstarke Schulen für unsere Kinder in Baden-Württemberg. Bildungskonzept Anlage. http://fraktion.cdu-bw.de/fileadmin/user_upload/infothek/ Bildung/2013-11-26_PM_216_Hauk_und_Wacker_zu_Bildungskonzept_ANLAGE_ Konzept.pdf. Zugegriffen: 05. Apr. 2016.

CDU. 2014. Eckpunktepapier Inklusion. Stuttgart: CDU-Landtagsfraktion. http://fraktion. cdu-bw.de/fileadmin/user_upload/infothek/Bildung/2014-07-31_Inklusionskonzept.pdf. Zugegriffen: 05. Apr. 2016.

CDU. 2015. Inklusion muss richtig gemacht werden! Rede von Dr. Monika Stolz MdL. http://fraktion.cdu-bw.de/fileadmin/user_upload/infothek/Bildung/2015-07-16_Plenarrede_Top_8_Dr._Stolz.pdf. Zugegriffen: 05. Apr. 2016.

CDU. 2016. Gemeinsam. Zukunft. Schaffen. Das Regierungsprogramm der CDU BadenWürttemberg 2016-2021. Stuttgart: CDU.

Die Grünen. 2011a. Das neue Programm für Baden-Württemberg: Jetzt. Stuttgart: Die Grünen.

Die Grünen. 2016. Grün aus Verantwortung für Baden-Württemberg: Innovative Wirtschaft - Gesunde Natur - Starke Familen - Offene Bürgergesellschaft. Wahlprogramm zu Landtagswahl 2016. Stuttgart: Die Grünen.

Die Grünen. 2011a. Grün-Rotes Bildungskonzept: Gute Schule entsteht von unten. https:// www.gruene-bw.de/gruen-rotes-bildungskonzept-gute-schule-entsteht-von-unten/. Zugegriffen: 05. Apr. 2016.

Die Grünen \& SPD. 2011. Der Wechsel beginnt. Koalitionsvertrag zwischen Die Grünen und der SPD Baden-Württemberg. Stuttgart: Die Grünen und SPD.

Edelstein, B., und R. Nikolai. 2013. Strukturwandel im Sekundarbereich. Determinanten schulpolitischer Reformprozesse in Sachsen und Hamburg. Zeitschrift für Pädagogik 59 (4): 482-494.

Expertenrat „Herkunft und Bildungserfolg“. 2011. Empfehlungen für Bildungspolitische Weichenstellungen in der Perspektive auf das Jahr 2020. Stuttgart: Ministerium für Kultus, Jugend und Sport.

Openpetition. O.J. Zukunft - Verantwortung - Lernen: Kein Bildungsplan unter der Ideologie des Regenbogens. https://www.openpetition.de/petition/online/zukunft-verantwortung-lernen-kein-bildungsplan-2015-unter-der-ideologie-des-regenbogens. Zugegriffen: 05. Apr. 2016.

FDP. 2010. Landeshauptausschuss am 09.10.2010 in Freudenstadt: Bildungsland BadenWürttemberg. http://www.fdp-bw.de/docs/Beschluss_Bildungsland_BW.pdf. Zugegriffen: 05. Apr. 2016.

FDP. 2011. Vorn bleiben. Erfolgsmodell Baden-Württemberg. Regierungsprogramm 2011 2016. Stuttgart: FDP Landesverband Baden-Württemberg.

FDP. 2012. Kern: Grün-Rot kommt bei der Inklusion nicht in die Gänge. http://fdp-dvpfraktion.de/pressemitteilungen/kern-grn-rot-kommt-bei-der-inklusion-nicht-in-diegnge/?a=1. Zugegriffen: 05. Apr. 2016. 
FDP. 2013. Kern: Grün-Rot muss sich bei Inklusion den Vorwurf der unterlassenen Hilfestellung gefallen lassen. http://fdp-dvp-fraktion.de/pressemitteilungen/kerngruen-rot-muss-sich-bei-inklusion-den-vorwurf-der-unterlassenen-hilfeleistung-gefallen-lassen/?a=1. Zugegriffen: 05. Apr. 2016.

FDP. 2014a. Impulspapier: Für mehr Freiheit und Eigenverantwortung in unserem Bildungswesen. Ein liberales Schulkonzept als Diskussionsgrundlage für einen stabilen Schulfrieden in Baden-Württemberg. http://fdp-dvp.de/downloads/ein-liberales-schulkonzept-als-diskussionsgrundlage-fuer-einen-stabilen-schulfrieden-in-baden-wuerttemberg.pdf. Zugegriffen: 05. Apr. 2016.

FDP. 2014b. Kern: Auch bei der Inklusion zeigt sich, dass Grün-Rot kein echtes Interesse an einem Schulfrieden hat. http://fdp-dvp-fraktion.de/pressemitteilungen/kern-auch-beider-inklusion-zeigt-sich-dass-gruen-rot-kein-echtes-interesse-an-einem-schulfriedenhat $/ ? \mathrm{a}=1$. Zugegriffen: 05. Apr. 2016.

FDP. 2016. Der nächste Schritt für unser Land. Das Wahlprogramm der Freien Demokraten Baden-Württemberg zur Landtagswahl 2016. Stuttgart: FDP Landesverband Baden-Württemberg.

Gemeindetag. 2012. Gesetzentwurf zur Gemeinschaftsschule mit gravierendem Mangel: Aussagen zu Kostenfolgen fehlen vollständig - Gemeindetag steht unverändert zum Schulversuch. https://www.gemeindetag-bw.de/internet/presse/pressemitteilungen-2012. Zugegriffen: 05. Apr. 2016.

Gemeindetag. 2013a. Forderung nach sofortiger regionaler Schulentwicklung bleibt bestehen - Gemeindetag begrüßt höhere Flexibilität bei Genehmigung von Gemeinschaftsschulen. https://www.gemeindetag-bw.de/internet/presse/pressemitteilungen-2013. Zugegriffen: 05. Apr. 2016.

Gemeindetag. 2013b. Regionale Schulentwicklung darf kein Papiertiger bleiben - Zumeldung des Gemeindetags zur Pressemitteilung des Staatsministeriums Landeskabinett gibt Gesetzentwurf zur regionalen Schulentwicklung zur Anhörung frei. https://www. gemeindetag-bw.de/internet/presse/pressemitteilungen-2013. Zugegriffen: 05. Apr. 2016.

Gemeindetag. 2015. Vereinbarung zwischen Land und Kommunen ist eine gute Grundlage für den Start der schulischen Inklusion. https://www.gemeindetag-bw.de/internet/ presse/pressemitteilungen-2015. Zugegriffen: 05. Apr. 2016.

Gingrich, J. R. 2011. Making markets in the welfare state: The politics of varying market reforms. Cambridge: Cambridge University Press.

Gingrich, J., und S. Häusermann. 2015. The decline of the working class vote, the reconfiguration of the welfare support coalition and consequences for the welfare state. Journal of European Social Policy 25:50-75.

Greif, A., und D.D. Laitin. 2004. A theory of endogenous institutional change. American Political Science Review 98 (4): 633-652.

Hanushek, E. A., und L. Wößmann. 2006. Does educational tracking affect performance and inequality? Differences-in-differences evidence across countries. Economic Journal 116:C63-C76.

Häusermann, S., G. Picot, und D. Geering. 2013. Review article: Rethinking party politics and the welfare state - recent advances in the literature. British Journal of Political Science 43 (1): 221-240. 
Helbig, M., und R. Nikolai. 2015. Die Unvergleichbaren: Der Wandel der Schulsysteme in den deutschen Bundesländern seit 1949. Bad Heilbrunn: Verlag Julius Klinkhardt.

Jacobs, A. M., und R. K. Weaver. 2015. When policies undo themselves: Self-undermining feedback as a source of policy change. Governance 28 (4): 441-457.

Kitschelt, H. 1994. The transformation of European social democracy. Cambridge: Cambridge University Press.

Kitschelt, H. 1999. European social democracy between political economy and electoral competition. In Continuity and change in contemporary capitalism, Hrsg. H. Kitschelt, P. Lange, G. Marks, und J.D. Stephens, 317-345. Cambridge: Cambridge University Press.

Köller, O., M. Knigge., und B. Tesch. Hrsg. 2010. Sprachliche Kompetenzen im Ländervergleich: Befunde des ersten Ländervergleichs zur Überprüfung der Bildungsstandards für den Mittleren Schulabschluss in den Fächern Deutsch, Englisch und Französisch Zusammenfassung https://www.iqb.hu-berlin.de/laendervergleich/LV08_09. Zugegriffen: 05. Apr. 2016.

Kultusministerium. 2010. Empfehlungen des Expertenrats zur schulischen Bildung von jungen Menschen mit Behinderung werden konsequent umgesetzt/Politische Beratung. http://www.kultusportal-bw.de/,Lde/778201/?LISTPAGE=778093. Zugegriffen: 05. Apr. 2016.

Kultusministerium. 2011a. Studie: Mehr Bildungsgerechtigkeit durch verbindliche Grundschulempfehlung. http://www.kultusportal-bw.de/,Lde/777625/?LISTPAGE=777557. Zugegriffen: 05. Apr. 2016.

Kultusministerium, 2011b. Kultusministerin besucht Landesschülerbeirat. http://www.kultusportal-bw.de/,Lde/777985/?LISTPAGE=777557. Zugegriffen: 05. Apr. 2016.

Kultusministerium. 2012. Kultusministerium: Inklusion hat einen überaus wichtigen politischen Stellenwert. http://www.kultusportal-bw.de/,Lde/777357/?LISTPAGE=776885. Zugegriffen: 05. Apr. 2016.

Kultusministerium. 2013a. Kultusministerin Gabriele Warminski-Leitheußer erklärt. http:// www.kultusportal-bw.de/,Lde/776841/?LISTPAGE=776833. Zugegriffen: 05. Apr. 2016.

Kultusministerium. 2013b. Landespräsident vereidigt neuen Kultusminister. http://www. kultusportal-bw.de/,Lde/776869/?LISTPAGE=776833. Zugegriffen: 05. Apr. 2016.

Kultusministerium. 2013c. Dazu-Meldung: Philologenverband redet Situation an Gymnasien schlecht - Aufruf: Rückkehr zur Sachpolitik. http://www.kultusportal-bw.de/,Lde/ Startseite/Bereich_Kommunikation/Zumeldung +PhV+_Zahlen+des+Philologenverba nds+nicht+nachvollziehbar_/?LISTPAGE=776833. Zugegriffen: 05. Apr. 2016.

Kultusministerium. 2015. Ministerrat gibt Gesetzentwurf zur Inklusion zur Anhörung frei. http://www.km-bw.de/,Lde/Startseite/Service/24_02_2015+MP+Inklusion/?LISTP $\mathrm{AGE}=3728528$. Zugegriffen: 05. Apr. 2016.

Kultusministerium. 2016. 28 weitere Gemeinschaftsschulen im Schuljahr 2016/17. http:// www.km-bw.de/,Lde/Startseite/Service/01_02_2016+Gemeinschaftsschulen+Start+zu m+Schuljahr+2016_2017. Zugegriffen: 05. Apr. 2016.

Landesinstitut für Schulentwicklung. 2015. Bildungsberichterstattung 2015: Bildung in Baden-Württemberg. Stuttgart: Landesinstitut für Schulentwicklung und Statistisches Landesamt Baden-Württemberg. 
LEB. 2011. Stellungnahme zum neuen Übergangsverfahren von der Grundschule in die weiterführenden Schulen. http://www.leb-bw.de/infos-downloads/stellungnahmen/ stellungnahmen-2011/105-stellungnahme-zur-grundschulempfehlung-und-kooperationmit-weiterf-schulen/file. Zugegriffen: 05. Apr. 2016.

LEB. 2012. Stellungnahme des 16. LEB zum Gesetzentwurf zur Einrichtung der Gemeinschaftsschule. http://www.leb-bw.de/infos-downloads/stellungnahmen/stellungnahmen2012/128-stellungnahme-des-leb-zur-gemeinschaftsschule/file. Zugegriffen: 05. Apr. 2016.

LEB. 2014. Stellungnahme des 16. Landeselternbeirates zum Gesetz zur Änderung des Schulgesetzes bezüglich der regionalen Schulentwicklung. http://www.leb-bw.de/infosdownloads/stellungnahmen/stellungnahmen-2014/237-stellungnahme-zur-regionalenschulentwicklung/file. Zugegriffen: 05. Apr. 2016.

Maier, S. 2015. Demo gegen Bildungsplan - Austragungsort eines Kulturkampfes. Stuttgarter Nachrichten, 22.06.2015. http://www.stuttgarter-nachrichten.de/inhalt. demo-gegen-bildungsplan-austragungsort-eines-kulturkampfs.dfac3a14-d867-4ddbacc0-6d1f4abf7377.html. Zugegriffen: 05. Apr. 2016.

Nikolai, R. 2007. Die Bildungsausgaben der Schweiz im intranationalen und internationalen Vergleich. Berlin: dissertation.de.

o. A. 2011. Deutliches Ja für eine „Schule von unten“. Stuttgarter Zeitung, 26.02.2011. http://www.genios.de/presse-archiv/artikel/STZ/20110226/deutliches-ja-fuer-eineschule-von-/BF176527C792FAA149EC515E8ECAD9BC.html. Zugegriffen: 05. Apr. 2016.

Osel, J. 2016. In letzter Sekunde. Süddeutsche Zeitung, 30.03.2016. http://www.sueddeutsche.de/bildung/baden-wuerttemberg-in-letzter-sekunde-1.2927271. Zugegriffen: 05. Apr. 2016.

otr/dpa. 2012. Baden Württemberg: Kretschmann will 11.600 Lehrer-Stellen streichen. http://www.spiegel.de/schulspiegel/baden-wuerttemberg-kretschmann-will-lehrerstellen-streichen-a-843705.html. Zugegriffen: 05. Apr. 2016.

Pfeffer, F.T. 2008. Persistent inequality in educational attainment and its institutional context. European Sociological Review 24(5): 543-565.

PHV. 2012. Einführung der Gemeinschaftsschule: Anhörungsentwurf zur Gesetzesänderung. http://www.phv-bw.de/Veroeffentlichung/Publikationen/GBW_2012_03/06gesetz.html. Zugegriffen: 05. Apr. 2016.

PHV. 2013. Hoher Anteil an überforderten Kindern in den fünften Klassen der Gymnasien. http://www.phv-bw.de/Veroeffentlichung/Pressemitteilungen/2013/pm_23-13.pdf. Zugegriffen: 05. Apr. 2016.

PHV. 2014. Hoher \%satz an überforderten Kindern in den fünften und sechsten Klassen der Gymnasien. http://www.phv-bw.de/Veroeffentlichung/Pressemitteilungen/2014/ pm_12-14.pdf. Zugegriffen: 05. Apr. 2016.

PHV. 2016. Junge Philologen fordern spürbare Verbesserungen der Arbeitsbedingungen für gymnasiale Lehrkräfte an Gemeinschaftsschulen. http://www.phv-bw.de. Zugegriffen: 05. Apr. 2016.

Pierson, P. 1993. When effect becomes cause: Policy feedback and political change. World Politics 45 (4): 595-628.

Pierson, P. 1994. Dismantling the welfare state? Reagan, thatcher, and the politics of retrenchment. Cambridge: Cambridge University Press. 
Pierson, P. 1996. The new politics of the welfare state. World Politics 48:143-179.

Pierson, P. Hrsg. 2001. The new politics of the welfare state. Oxford: Oxford University Press.

Preuss-Lausitz, U. 2010. Inklusion aus Sicht der Erziehungswissenschaft - Vorschläge zur landesweiten Umsetzung inklusiver Unterrichts- und Schulentwicklung. Vortrag im Rahmen des SPD-Forums „Schule ist für alle da - das Recht auf Inklusion“. http://bildungsaufbruch.bawue.spd.de/cgi-sub/fetch.php?id=108. Zugegriffen: 05. Apr. 2016.

Rauh, C., A. Kirchner, und R. Kappe. 2011. Political parties and higher education spending: Who favours redistribution? West European Politics 34 (6): 1185-1206. doi:10.10 80/01402382.2011.616659. Zugegriffen: 05. Apr. 2016.

red/dpa. 2016. Reformen bis 2020: Kretschmann sagt Schulen zusätzliche Lehrerstellen zu. Stuttgarter Nachrichten, 04.03.2016. http://www.stuttgarter-nachrichten.de/inhalt. reformen-bis-2020-kretschmann-sagt-schulen-zusaetzliche-lehrerstellen-zu.e071a3133ce6-4df7-9fbd-74fd3d8de9ec.html. Zugegriffen: 05. Apr. 2016.

RLV. 2012. Zumeldung des Realschullehrerverbandes (RLV) Baden-Württemberg zur Pressemitteilung des Kultusministeriums Nr. 106/2012. Wahlbetrug oder die Mär von der individuellen Förderung an der Realschule? http:/www.rlv-bw.de/fileadmin/content/ PDFs/presse/pressemitteilungen/008_PM-07092012-Wahlbetrug-oder.pdf. Zugegriffen: 05. Apr. 2016.

RLV. 2013. Schluss mit der ideologisch gesteuerten Bildungspolitik! RLV fordert erneut Anpassung der Sachkostenbeiträge und Lehrerstunden zur individuellen Förderung. http://www.rlv-bw.de/fileadmin/content/PDFs/presse/pressemitteilungen/021_PM07052013-Kuerzungen-AE.pdf. Zugegriffen: 05. Apr. 2016.

Rodenhausen, F. 2010. Der Weg zur gemeinsamen Bildung. Stuttgarter Zeitung, 09.08.2010. http://www.stuttgarter-zeitung.de/inhalt.der-weg-zur-gemeinsamen-bildung.057c83a93fd8-40f4-b712-ed0eb8b86618.html. Zugegriffen: 05. Apr. 2016.

Rose, R., und P. L. Davies. 1994. Inheritance in public policy: Change without choice in Britain. Yale: Yale University Press.

Schlauch, R., und R. Weber. 2015. Keine Angst vor der Macht: Die Grünen in Baden-Württemberg. Köln: Emons Verlag.

Schmidt, M. G. 1980. CDU und SPD an der Regierung: Ein Vergleich ihrer Politik in den Ländern. Frankfurt a. M.: Campus Verlag.

Schmidt, M. G. 1996. When parties matter: A review of the possibilities and limits of partisan influence on public policy. European Journal of Political Research 30 (2): 155-183.

Schmidt, M. G. 2002. The impact of political parties, constitutional structures and veto players on public policy. In Comparative democratic politics: A guide to contemporary theory and research, Hrsg. H. Keman, 166-184. London: Sage.

Schmidt, M.G. 2007. Testing the retrenchment hypothesis: Educational spending, 1960 2002. In The disappearing state? Retrenchment realities in an age of globalisation, Hrsg. F. G. Castles, 159-183. Cheltenham: Edward Elgar.

Schwarz-Jung, S. 2014. Die Gemeinschaftsschule: Eine neue Schulart in BadenWürttemberg. Statistisches Monatsheft Baden-Württemberg 5, 5-11.

Soroka, S. N., und C. Wlezien. 2010. Degrees of democracy: Politics, public opinion, and policy. Cambridge: Cambridge University Press.

SPD. 2008. Bildungsaufbruch für alle. Flyer zur Bildungskampagne der SPD-Landtagsfraktion. http://bildungsaufbruch.bawue.spd.de/cgi-sub/fetch.php?id=79. Zugegriffen: 05. Apr. 2016. 
SPD. 2011. Regierungsprogramm der SPD Baden-Württemberg 2011-2016. Stuttgart: SPD.

SPD. 2015. Bildungsbericht unterstreicht Notwendigkeit regionaler Schulentwicklung. https://www.spd-bw.de/index.php?nr=89867\&menu=1. Zugegriffen: 05. Apr. 2016.

SPD. 2016. Baden-Württemberg leben. Regierungsprogramm der SPD Baden-Württemberg 2016-2021. Stuttgart: SPD.

Städtetag. 2011. Schaffung stabiler und verlässlicher Schulstrukturen muss oberstes Bildungsziel des Landes sein. http://www.staedtetag-bw.de/media/custom/1198_62358_1. PDF?1323240695. Zugegriffen: 05. Apr. 2016.

Städtetag. 2012. Gesetz zur Gemeinschaftsschule; Anhörung. http://www.staedtetag-bw.de/ media/custom/1198_63569_1.PDF?1328271742. Zugegriffen: 05. Apr. 2016.

Städtetag. 2013. Regionale Schulentwicklung, Gemeinschaftsschule und Ganztagsschule im Städtetagsschulausschuss mit Kultusminister Stoch MdL. http://www.staedtetag-bw. de/media/custom/safe/1198_75104_1.PDF?1364206822. Zugegriffen: 05. Apr. 2016.

Städtetag. 2015. Inklusive Bildungsangebote in Baden-Württemberg; Anhörung zur Änderung des Schulgesetzes für BW und anderer Vorschriften. http://www.staedtetag-bw.de/ media/custom/2295_12199_1.PDF?1426686608. Zugegriffen: 05. Apr. 2016.

Stoch, A. 2016. Vorwort. Bildungspläne 2016. http://www.bildungsplaene-bw.de/,Lde/ Startseite. Zugegriffen: 05. Apr. 2016.

Streeck, W., und K. Thelen. 2005. Introduction: Institutional change in advanced political economies. In Beyond continuity: Institutional change in advanced political economies, Hrsg. W. Streeck und K. Thelen, 1-39. Oxford: Oxford University Press.

VBE. 2012. VBE lobt die Grundschule: „Ein“ „richtiges Erfolgsmodell“. http://www.vbebw.de/grundschule/vbe-lobt-die-grundschule-ein-richtiges-erfolgsmodell-2/. Zugegriffen: 05. Apr. 2016.

Von Below, S. 2002. Bildungssysteme und soziale Ungleichheit: Das Beispiel der neuen Bundesländer. Opladen: Leske \& Budrich.

Weaver, R.K. 2010. Paths and forks or chutes and ladders?: Negative feedbacks and policy regime change. Journal of Public Policy 30 (2): 137-162.

Wieselmann, B. 2013. Bildungspolitik - Kultusministerin wirft hin. Der Tagesspiegel, 08.01.2013. http://www.tagesspiegel.de/wissen/bildungspolitik-kultusministerin-wirfthin/7597746.html. Zugegriffen: 05. Apr. 2016.

Wilms, D. 1986. Bildungspolitik im Umbruch. In Berufsbildung 2000, DIHT-Jubiläum in Ludwigshafen, 30. September 1986, Hrsg. H.-J. Demuth, 9-24. Bonn: Deutscher Industrie- und Handelstag.

Wolf, F. 2006. Die Bildungsausgaben der Bundesländer im Vergleich: Welche Faktoren erklären ihre beträchtliche Variation?. Münster: LIT Verlag.

Wolf, F. 2008. Die Schulpolitik - Kernbestand der Kulturhoheit. In Die Schulpolitik Kernbestand der Kulturhoheit, Hrsg. F. Wolf, 21-41. Wiesbaden: VS Verlag.

Wolf, F., und R. Zohlnhöfer. 2009. Investing in human capital? The determinants of private education expenditure in 26 OECD countries. Journal of European Social Policy 19 (3): 230-244. 


\section{Über die Autoren}

Prof. Dr. Marius R. Busemeyer ist Inhaber des Lehrstuhls für Politikwissenschaft, insbesondere Policy-Analyse und Politische Theorie, an der Universität Konstanz. Seine Forschungsschwerpunkte sind die vergleichende politische Ökonomie, Bildungspolitik im internationalen Vergleich, Wohlfahrtsstaaten, Staatsfinanzen sowie öffentliche Meinung zu Bildungs- und Sozialpolitik.

Susanne Haastert ist akademische Mitarbeiterin und Doktorandin am Lehrstuhl für Politikwissenschaft, insbesondere Policy-Analyse und Politische Theorie, der Universität Konstanz. In ihrer Forschung befasst sie sich schwerpunktmäßig mit Bildungssystemen, Bildung und politischer Partizipation in Demokratien und Autokratien. 